\title{
Reprogramming metabolism by targeting sirtuin 6 attenuates retinal degeneration
}

\author{
Lijuan Zhang, ${ }^{1,2,3}$ Jianhai Du, ${ }^{4}$ Sally Justus, ${ }^{1,2}$ Chun-Wei Hsu, ${ }^{1,2}$ Luis Bonet-Ponce, ${ }^{5}$ Wen-Hsuan Wu, ${ }^{1,2}$ Yi-Ting Tsai, ${ }^{1,2}$ Wei-Pu Wu, ${ }^{1,2}$ \\ Yading Jia, ${ }^{2}$ Jimmy K. Duong, ${ }^{6}$ Vinit B. Mahajan, ${ }^{7}$ Chyuan-Sheng Lin, ${ }^{8}$ Shuang Wang, ${ }^{6}$ James B. Hurley, ${ }^{4}$ and Stephen H. Tsang ${ }^{1,2,9}$ \\ 'The Jonas Children's Vision Care and Bernard \& Shirlee Brown Claucoma Laboratory, Department of Ophthalmology, Columbia University, New York, New York, USA. ${ }^{2}$ Edward S. Harkness Eye Institute, \\ New York-Presbyterian Hospital, New York, New York, USA. ${ }^{3}$ Shanxi Eye Hospital, affiliated with Shanxi Medical University, Xinghualing, Taiyuan, China. ${ }^{4}$ Department of Biochemistry and Ophthalmology, \\ University of Washington, Seattle, Washington, USA. ${ }^{5}$ Section on Retinal Ganglion Cell Biology, Laboratory of Retinal Cell and Molecular Biology, NIH, Bethesda, Maryland, USA. ${ }^{6}$ Department of Biostatistics, \\ Mailman School of Public Health, Columbia University Medical Center, New York, New York, USA. `Omics Lab, University of lowa, lowa City, lowa, USA. ${ }^{8}$ Department of Pathology and Cell Biology, Herbert \\ Irving Comprehensive Cancer Center, College of Physicians and Surgeons of Columbia University, New York, New York, USA. ${ }^{9}$ Departments of Pathology and Cell Biology, Institute of Human Nutrition, College \\ of Physicians and Surgeons, Columbia University, New York, New York, USA.
}

\begin{abstract}
Retinitis pigmentosa (RP) encompasses a diverse group of Mendelian disorders leading to progressive degeneration of rods and then cones. For reasons that remain unclear, diseased RP photoreceptors begin to deteriorate, eventually leading to cell death and, consequently, loss of vision. Here, we have hypothesized that RP associated with mutations in phosphodiesterase-6 (PDE6) provokes a metabolic aberration in rod cells that promotes the pathological consequences of elevated cGMP and $\mathrm{Ca}^{2+}$, which are induced by the Pde6 mutation. Inhibition of sirtuin 6 (SIRT6), a histone deacetylase repressor of glycolytic flux, reprogrammed rods into perpetual glycolysis, thereby driving the accumulation of biosynthetic intermediates, improving outer segment (OS) length, enhancing photoreceptor survival, and preserving vision. In mouse retinae lacking Sirt6, effectors of glycolytic flux were dramatically increased, leading to upregulation of key intermediates in glycolysis, TCA cycle, and glutaminolysis. Both transgenic and AAV2/8 gene therapy-mediated ablation of Sirt6 in rods provided electrophysiological and anatomic rescue of both rod and cone photoreceptors in a preclinical model of RP. Due to the extensive network of downstream effectors of Sirt6, this study motivates further research into the role that these pathways play in retinal degeneration. Because reprogramming metabolism by enhancing glycolysis is not gene specific, this strategy may be applicable to a wide range of neurodegenerative disorders.
\end{abstract}

\section{Introduction}

Retinitis pigmentosa (RP) encompasses a diverse group of inherited genetic disorders that lead to progressive photoreceptor degeneration, night blindness, tunnel vision, and, eventually, loss of the ability to conduct many activities of daily living. One of the most severe retinopathies, $\mathrm{RP}$ is estimated to affect 1.5 million people worldwide, and 1 in 10 Americans carries a recessive RP allele (1), including mutations in phosphodiesterase-6 (PDE6), a G protein effector that controls cGMP levels (2-7). Rod photoreceptor cells are affected first, and accordingly, a patient's first symptom typically is poor night vision, followed by tunnel vision. The massive loss of rod cells is associated with the secondary death of cone photoreceptors (8). After the loss of a significant numbers of cone cells, patients usually go blind. Subsequently, retinal pigment epithelium (RPE) cells, which support and maintain photoreceptors, migrate into the outermost layer of the retina, where they also experience cell death (8).

While $\mathrm{RP}$ is a genetically heterogeneous disorder, more than $8 \%$ of all autosomal recessive RP cases arise from mutations in the PDE6 gene (9). This prompted the creation of a mouse line that mimics this

Conflict of interest: The authors have declared that no conflict of interest exists. Submitted: February 4, 2016; Accepted: October 6, 2016.

Reference information: J Clin Invest. 2016;126(12):4659-4673. doi:10.1172/JCI86905. phenotype: the Pde6 mutant mouse is one of the most commonly studied models of RP. In this study, we used mice with a histidine to glutamine mutation (Pde6b ${ }^{\mathrm{H} 620 \mathrm{O} / \mathrm{H} 620 \mathrm{O}}$ ), which develop a severe form of RP where retinal degeneration begins as early as $2-3$ weeks after birth, and a near complete loss of photoreceptors is observed by 8 weeks (10).

Normally, the OS of photoreceptors are continuously regenerated in a process that requires NADPH from the pentose phosphate pathway (PPP) to generate phospholipids $(11,12)$. Rods shuttle glucose into the PPP to synthesize new membranes and facilitate OS generation $(13,14)$. In darkness, rods are constantly depolarized and consume more glucose than nearly all other cell types (15-20). However, in RP, an early manifestation of the disease is dysgenesis of rod OS (21). Photoreceptor death in the equatorial plane induces a bull's-eye pattern of cell loss that is common among RP patients (22).

Some success in the treatment of RP has been achieved using gene therapy. Clinical trials for a form of RP called Leber congenital amaurosis slowed photoreceptor death but did not halt degeneration (23-32). While there are ongoing efforts to improve this therapy, a limitation of gene-specific treatments is the vast genetic heterogeneity of RP, with more than 60 genes associated with the disorder (33-35). Efforts to identify non-gene-specific therapies have had promising results. Activation of the mammalian target of rapamycin complex 1 (mTORC1) prolonged cone survival for 8 
A
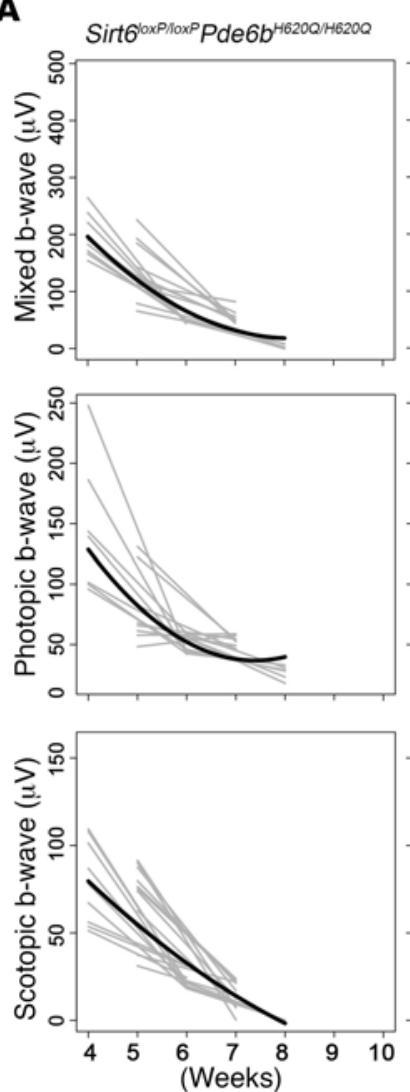

Sirt6 $\%$ Pde $6 b^{H 6200 / H 6200}$
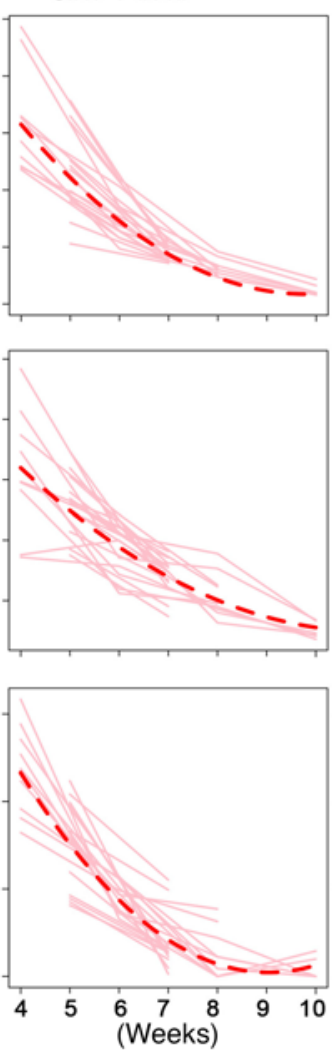
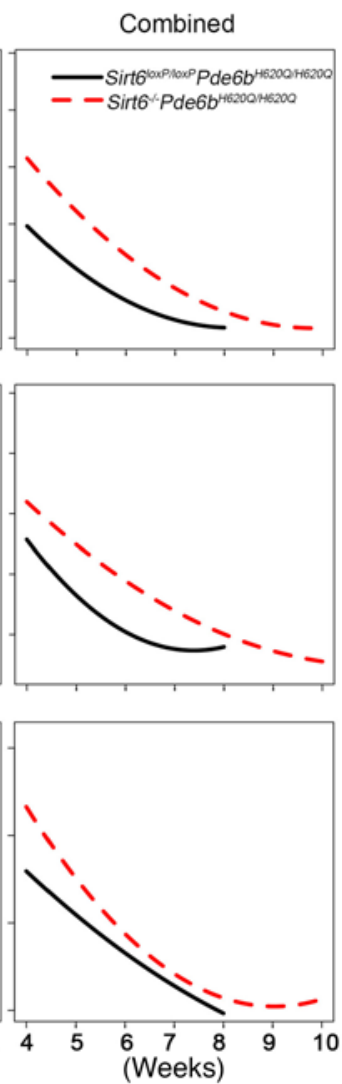

B
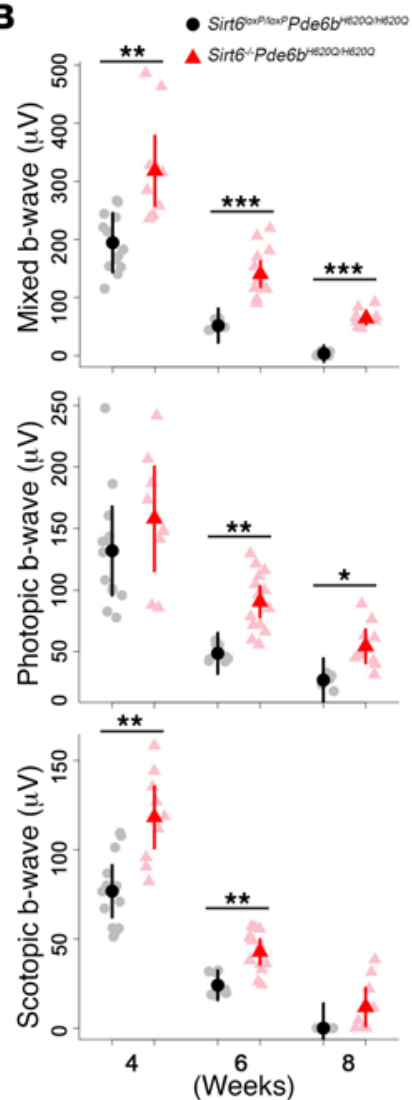

Figure 1. Sirt6 deficiency improves electrophysiological function in both rods and cones. (A) ERG was obtained weekly under dark- and light-adapted conditions to acquire scotopic, photopic, and mixed rod-cone b-wave amplitudes $(\mu \mathrm{V})$. Linear mixed models were fit to estimate the trajectory of outcomes over time, and differences were assessed by likelihood ratio tests. Gray and light red lines represent individual eyes; solid black and dashed red lines represent mean trajectories from the mixed models for Sirt6 ${ }^{10 x P / l o x P} P d e 6 b^{H 6200 / H 6200}$ and Sirt6 $\sigma^{-/-} P d e 6 b^{H 6200 / H 6200}$, respectively. Sirt6 ${ }^{-1-} P d e 6 b^{H 6200 / H 6200}$ mice had higher trajectories compared with control mice for each outcome: mixed $(P<0.001)$, photopic $(P=0.048)$, and scotopic $(P=0.004)$. For Sirt6 $6^{l 0 x P / l o x P} P d e 6 b^{H 6200 / H 6200}$ : at 4 weeks,

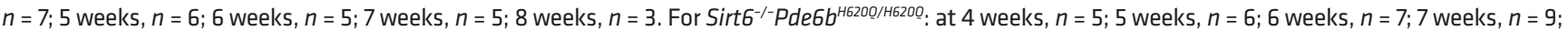
8 weeks, $n=5$; 10 weeks, $n=3$. (B) Comparison of ERG data under scotopic, photopic, and mixed conditions at 4, 6 , and 8 weeks. Gray dots and light red trian-

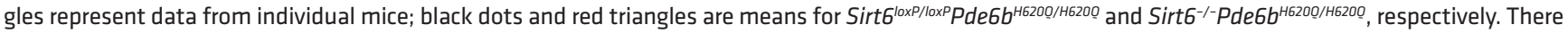
were significant differences between the groups at all time points and all light adaptation conditions except for photopic conditions at 4 weeks and scotopic at 8 weeks. A linear mixed model was fit to compare groups. At 4 weeks: scotopic b-wave $P=0.004$, mixed b-wave $P=0.01$, photopic b-wave $P=0.3$; 6 weeks: scotopic b-wave $P=0.007$, mixed b-wave $P<0.001$, photopic b-wave $P=0.003$; 8 weeks: scotopic b-wave $P=0.2$, mixed b-wave $P<0.001$, photopic b-wave $P=0.048$. ${ }^{*} P<0.05,{ }^{* *} P<0.01$, and ${ }^{* * *} P<0.001$.

months by increasing glucose uptake and utilization for NADPH production in 4 different mouse models of RP $(11,12)$. In our report, we propose a similar strategy that improves both survival and function of degenerating rods and cones. We hypothesized that Pde6-associated RP provokes a metabolic aberration in the rod cells that forces them to succumb to the consequences of elevated cGMP and $\mathrm{Ca}^{2+}$ via cyclic nucleotide-gated (CNG) channels and $\mathrm{Na}^{+} / \mathrm{Ca}^{2+}-\mathrm{K}^{+}$exchangers (36-39).

The histone deacetylase sirtuin 6 (SIRT6) is a transcriptional repressor of glycolytic enzymes that has been extensively studied in the context of metabolism and cancer biology (40). Normally, SIRT6 directs glucose metabolism to proceed through aerobic metabolism by maintaining histone $\mathrm{H} 3$ (H3K9) in a deacetylated form, thereby repressing hypoxia-inducible factor $1 \alpha$ (HIF1A), a transcription factor (41). When nutrients are scarce or when SIRT6 is systemically suppressed experimentally, glucose is preferentially processed through glycolytic pathways. We hypothesized that knockdown of SIRT6 in rod photoreceptors could theoretically remodel cellular metabolism to favor glycolysis over respiration (42-44). This should increase the concentrations of intermediates in glycolysis and the PPP, promoting survival. Previous studies of the role of Sirt6 in retinal function in a conventional systemic knockout, Sirt6 ${ }^{\text {tmIFwa }}$, were challenging to interpret because of negative effects on synaptic transmission (45). We therefore altered our approach to limit ablation of Sirt6 to rod photoreceptors with an inducible gene disruption strategy. Using this model, we tested whether upregulation of glycolytic flux through Sirt6 knockout can preserve both rod and cone photoreceptors in a preclinical, Pde6-associated RP model.

\section{Results}

Generation of experimental and control groups. The third most common cause of autosomal recessive RP is deficiency in the PDE6 enzyme, which controls the depolarization state of rods by regulating cGMP levels $(9,46-48)$. An established preclinical model for $\mathrm{RP}$ involves a homozygous point mutation (H620Q) in the gene 
A

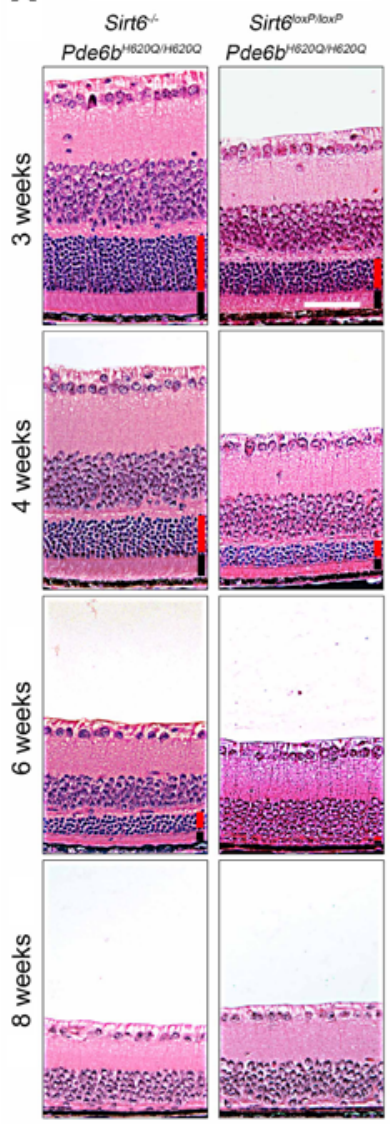

B

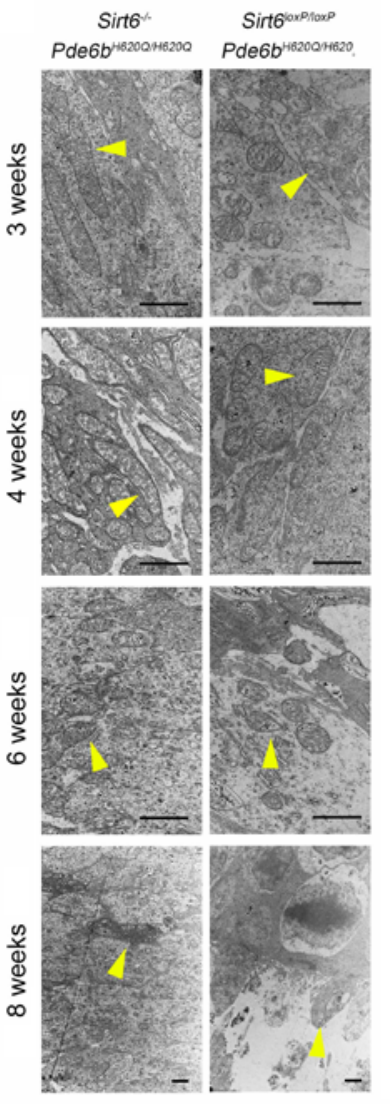

C

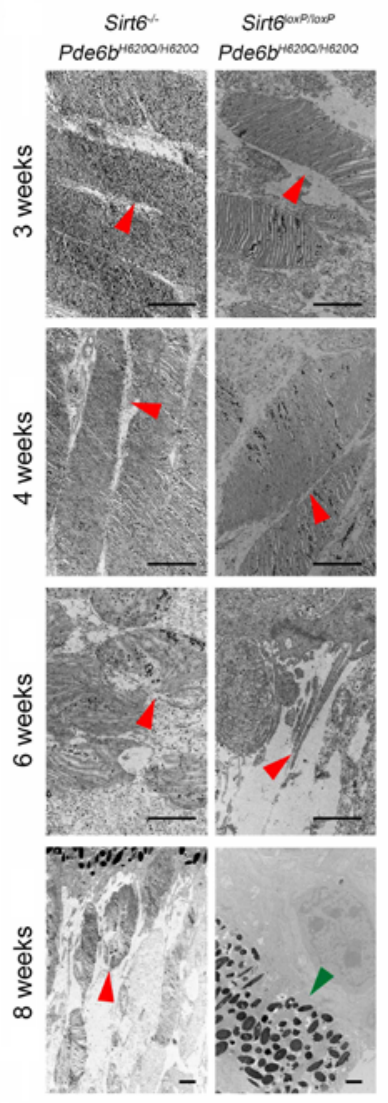

D

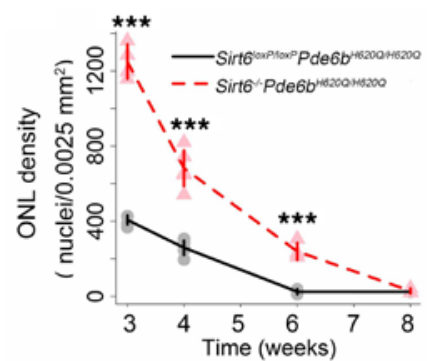

$\mathbf{E}$

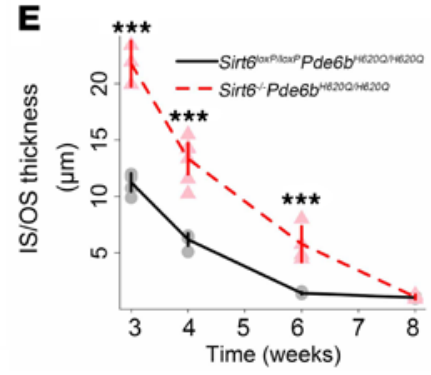

Figure 2. Sirt6 deficiency improves rod and cone survival and robustness. (A) H\&E staining of paraffin sections of retinae showed thicker ONL and IS/OS

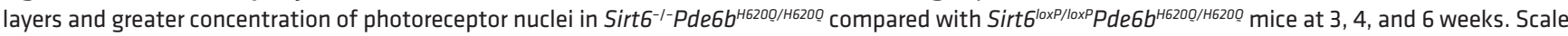
bar: $50 \mu \mathrm{m}$. Red bars represent ONL. Black bars represent IS/OS. (B) TEM reveals healthier mitochondria (yellow arrowheads) in treated mice, with more cristae and longer cell bodies in the Sirt6 ${ }^{-1-P d e 6} b^{H 6200 / H 6200}$ mice than the Sirt6 ${ }^{10 \times P / 10 \times P} P d e 6 b^{H 6200 / H 6200}$ mice. However, by 8 weeks, both groups' mitochondria appeared equally damaged. Scale bars: $1 \mu \mathrm{m}$. (C) TEM of OS (red arrowheads) of photoreceptors showed longer, more plentiful discs in Sirt6 ${ }^{-1-}$ Pde66 ${ }^{H 6200 / H 6200}$ compared with Sirt6 ${ }^{10 \times P / / 10 \times P} P d e 6 b^{H 6200 / 46200}$ mice. There was a decline over time in both groups, although by 8 weeks, the knockout group still retained detectable OS, while the control group appeared completely deficient in them. Green arrowhead points to the boundary between the RPE cells and the bipolar cells' nuclei. Scale bars: $1 \mu \mathrm{m}$. ( $\mathbf{D}$ and $\mathbf{E}$ ) Quantification of the ONL density (nuclei/0.0025 $\mathrm{mm}^{2}$ ) (D) and IS/OS thickness ( $\mu \mathrm{m}$ ) (E) corroborated histological findings, confirming that Sirt6-deficient mice had significantly higher density and thickness of the ONL and IS/OS layers, respectively. Two-tailed $t$ tests were used to analyze the data. ONL cell density at 3,4 , and 6 weeks $P<0.001 ;$ IS/OS thickness at 3 and 4 weeks, $P<0.001$, at 6 weeks $P=0.002 ; 8$ weeks, $P=0.6$ for

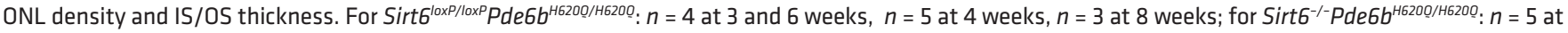
3 and 4 weeks; $n=4$ at 6 and 8 weeks. ${ }^{* * *} P<0.001$.

encoding the $\beta$ subunit of PDE6 (Pde6b), which causes delayed-onset RP. Photoreceptor degeneration begins when the mice are 2-3 weeks old, and by 8 weeks of age, nearly all photoreceptors have degenerated, and minimal functionality remains $(10,39)$. For gene therapy in this preclinical mouse model, we generated a tamoxifen-inducible rod photoreceptor-specific Cre recombinase to conditionally inactivate expression of the loxP-modified Sirt $6^{\text {tm.1.1Cxd }}$ gene. Experimental mice were bred from 3 homozygous strains: Pde6b ${ }^{H 620 Q}$, Pde6gCreERT2 and Sirt6 mice. Thus, the experimental mice were homozygous for Pde $6 b^{H 620 Q}$ and Sirt $6^{\text {tml.1Cxd }}$ and heterozygous for Pde6g $g^{\text {CreERT2 }}$. Half of the experimental offspring were injected with tamoxifen and served as the experimental group, henceforth referred to as Sirt6-deficient (Sirt6-/-Pde6b ${ }^{\mathrm{H} 620 \mathrm{O} / \mathrm{H} 620 \mathrm{O}}$ Pde6g ${ }^{\text {CreERT2 }}$ ) and denoted Sirt6 ${ }^{-1}$ Pde $6 b^{H 6200 / H 620 Q}$, whereas the other half were injected with oil and served as controls (Sirt $6^{\text {loxp/lox }}$ Pde6 $b^{\text {H620Q/H620QPde }}$ g $\left.^{\text {CreERT2 }}\right)$, denoted Sirt6 $6^{\text {loxP/loxP } P d e 6 ~} b^{H 620 Q / H 620 Q}$.
Upon tamoxifen induction, the Pde $6 g^{\text {CreERT2 }}$ recombinase excised exons 2 and 3 of the Sirt6 gene in the retinae of Sirt $6^{-1} \mathrm{Pde} 6 b^{\mathrm{H} 620 \mathrm{O} / \mathrm{H} 620 \mathrm{O}}$ mice. A 524-bp band was found in DNA amplified from the outer nuclear layer (ONL) of retinae collected from tamoxifen-treated mice, but not in DNA amplified from the ONL of the control mice (Supplemental Figure 1; supplemental material available online with this article; doi:10.1172/JCI86905DS1). A 2,048-bp nonrecombined product was also noted in other organs of both Sirt6- $\mathrm{Pde} 6 b^{\mathrm{H} 620 \mathrm{Q} / \mathrm{H} 620 \mathrm{Q}}$

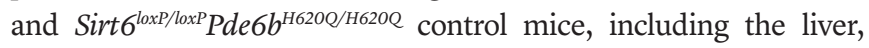
lungs, kidneys, and pancreas. This demonstrates the specificity of the Pde6g GreeRT2 recombinase for the retina and suggests that offtarget effects from the tamoxifen injection were limited.

Functional rescue of retinal electrophysiological properties in Sirt6-1-Pde6b ${ }^{\mathrm{H} 620 \mathrm{e} / \mathrm{H} 620 \mathrm{O}}$ mice. To assess functional rescue, we performed electroretinography (ERG) analysis weekly (Figure 1). The trend lines projected for the Sirt $6^{-/} P d e 6 b^{H 620 Q / H 620 Q}$ mice 
A
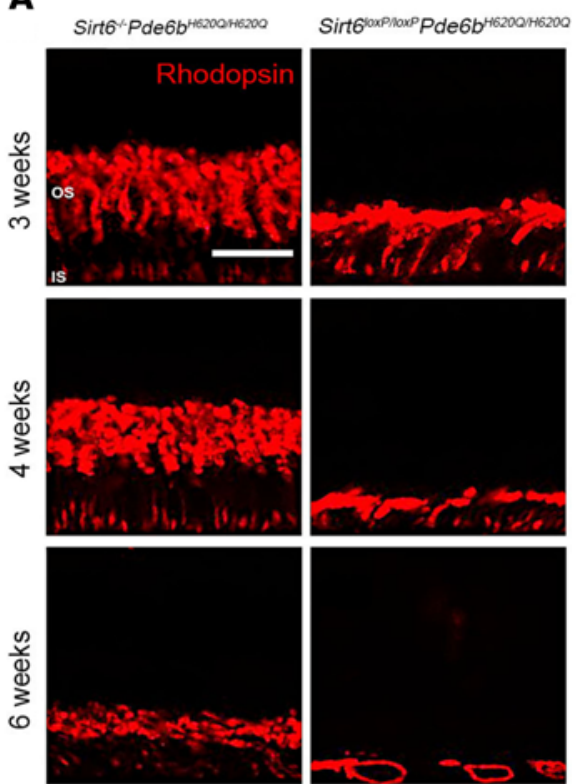

B

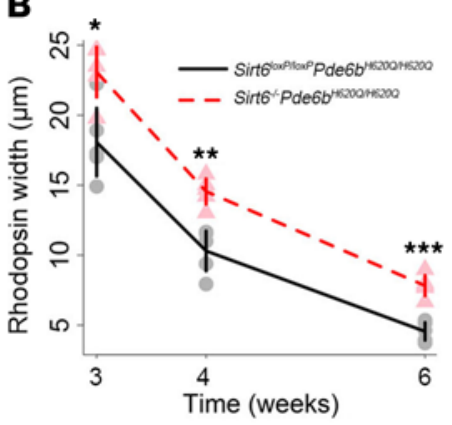

$\mathbf{F}$

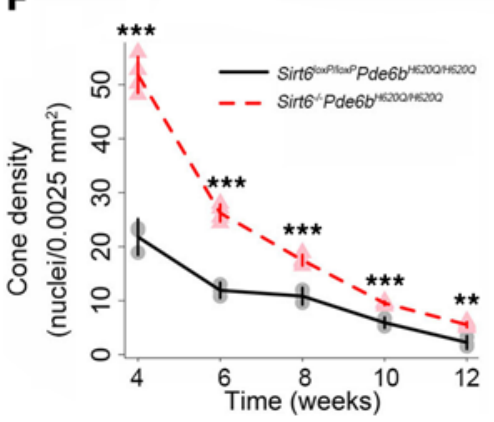

C
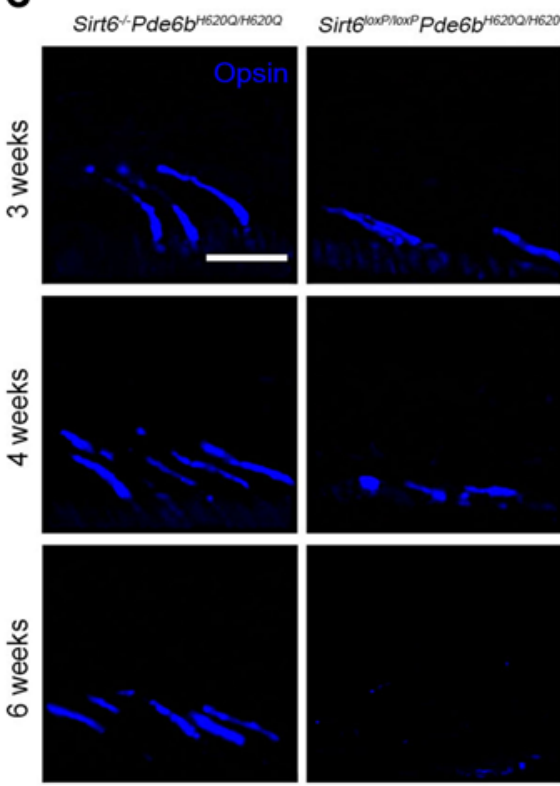

D
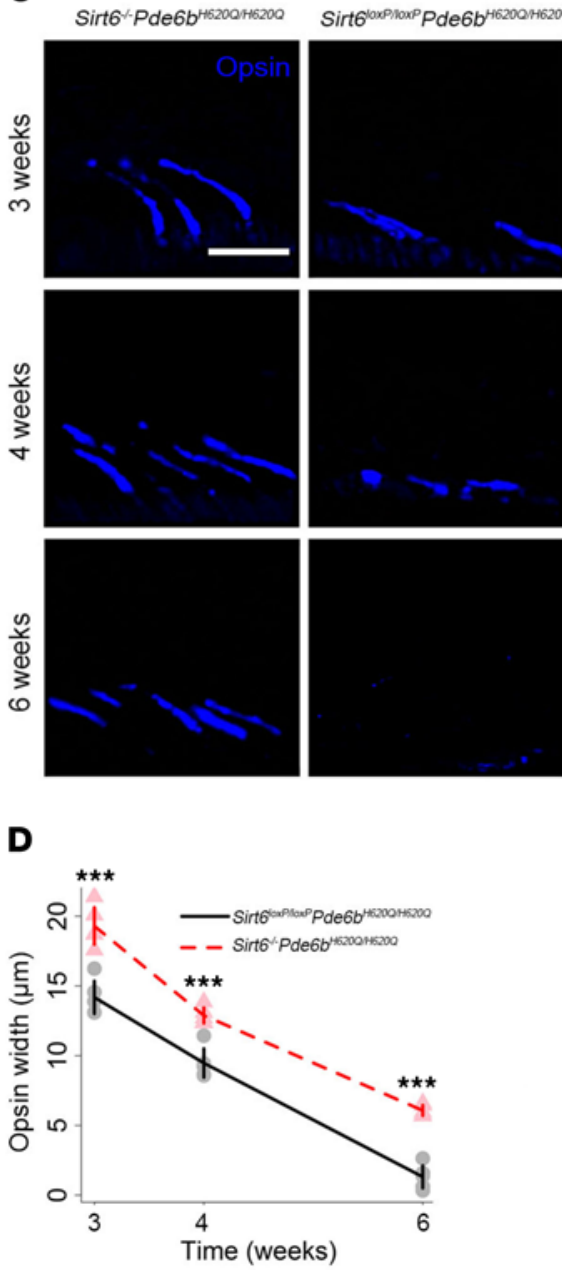

E
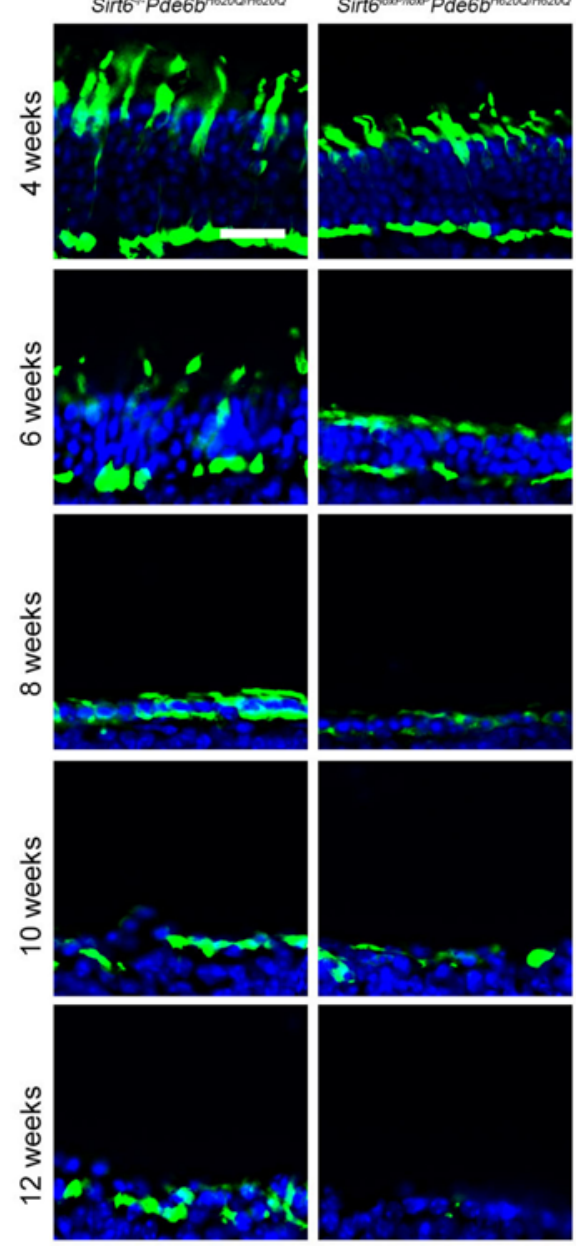

Figure 3. Sirt6 deficiency promotes photoreceptor survival and preserves cellular 0 S. (A and B) Rhodopsin antibody staining of retinal OS layers was used to compare morphological differences between control and treated mice. The expression of rhodopsin was greater in the Sirt6 ${ }^{-1-P d e 6 b^{H 6200 / H 6200}}$ mice at every time point. (B) Gray dots represent data from individual Sirt6 ${ }^{10 \times P / / 0 \times P} P d e 6 b^{H 6200 / H 6200}$ mice, and a black trend line was projected to connect the means at each time point. Light red triangles represent data from individual Sirt6 ${ }^{-1-}$ Pde6 $6 b^{H 6200 / 46200}$ mice, and the red dashed line represents a projected trendline to connect the means for the Sirt6 ${ }^{-1-} P d e 6 b^{H 6200 / H 6200}$ mice at each time point. Two-tailed $t$ tests were used to analyze the data. 3 weeks: $P=0.012 ; 4$ weeks: $P=0.002 ; 6$ weeks: $P<0.001 . n=5$ per group at all time points. Scale bars: $20 \mu \mathrm{m}$ throughout figure (A, C, and E). (C and D) Short-wavelength cone opsin antibody (blue) staining of retinal OS layers allowed comparison of morphological differences. The $\mathrm{OS}$ of cones were elongated in treated mice. (D) Graphical representations and statistical analyses are as indicated in $\mathbf{A}$ and $\mathbf{B}$. 3 weeks: $P<0.001 ; 4$ weeks: $P=0.001 ; 6$ weeks: $P<0.001 . n=5$ per group at all time points. (E and F) DAPI (blue) and anti-cone arrestin (green) staining allowed visualization of retina nuclei and cone cell markers, respectively. Results were merged into composite images. Cone density (nuclei/ $0.0025 \mathrm{~mm}^{2}$ ) was greater in the Sirt6-knockout mice at every time point compared with controls. (F) Graphical representations and statistical analyses are as indicated in $\mathbf{A}$ and $\mathbf{B} . P<0.001$ at $4,6,8$, and 10 weeks; $P=0.0016$ at 12 weeks. For Sirt6 ${ }^{10 \times P / / 10 \times P} P d e 6 b^{H 6200 / H 6200}: n=3$ at all time points; for Sirt6 ${ }^{-1-}$ Pde6b $b^{H 6200 / H 6200}: n=4$ at all time points. ${ }^{*} P<0.05,{ }^{*} P<0.01$, and ${ }^{* *} P<0.001$.

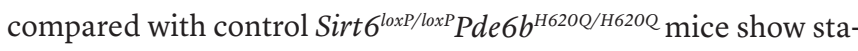
tistically significant differences in scotopic, photopic, and mixed ERG responses. Under dark-adapted conditions, the mixed and scotopic b-wave responses measuring the electrophysiological function of rod cells (Figure 1A, top and bottom) were significantly higher in Sirt6 ${ }^{-1} P d e 6 b^{H 620 Q / H 620 Q}$ mice compared with Sirt $6^{\text {loxP/loxP } P d e 6} b^{H 620 Q / H 620 Q}$ mice. Light-adapted conditions were used to measure the cone response and produced higher b-wave responses (Figure 1A, middle) in the Sirt6 ${ }^{-/-P d e} 6 b^{\mathrm{H} 620 \mathrm{Q} / \mathrm{H} 620 \mathrm{Q}}$ mice compared with controls. At 10 weeks, the scotopic, photopic, and mixed b-wave responses were not measurable in the Sirt $6^{\text {loxp } / \text { loxP }}$ Pde6 $b^{\mathrm{H} 620 \mathrm{Q} / \mathrm{H} 620 \mathrm{Q}}$ controls, whereas the Sirt6 ${ }^{-1-} \mathrm{Pde} 6 b^{\mathrm{H} 620 \mathrm{Q} / \mathrm{H} 620 \mathrm{Q}}$ mice retained measurable functionality.

We found that the greatest difference between the b-wave responses of the treated and control groups occurred at 4 weeks in rods under scotopic conditions (Figure 1B, bottom). This difference 
A

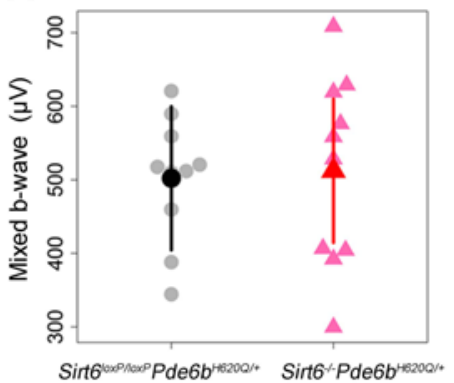

B

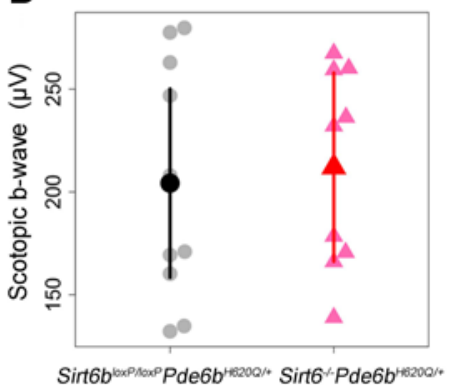

C

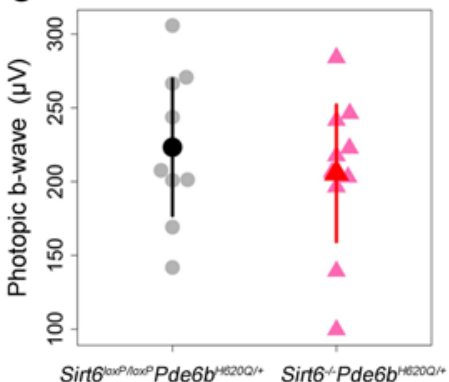

D

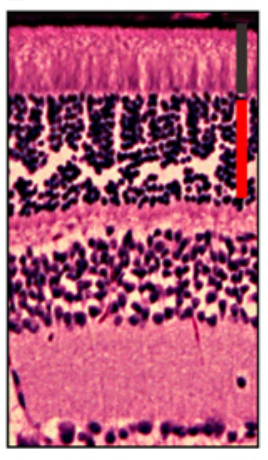

Sirt6 ${ }^{10 \times P / 10 \times P} P d e 6 b^{16200 /}$

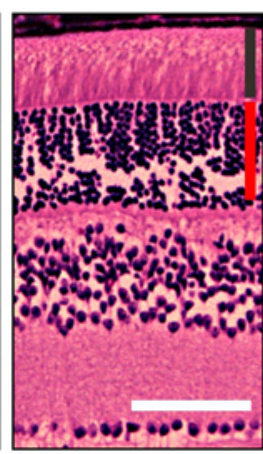

Sirt6 $\%$ Pde6 $6 b^{16200 / \%}$
E

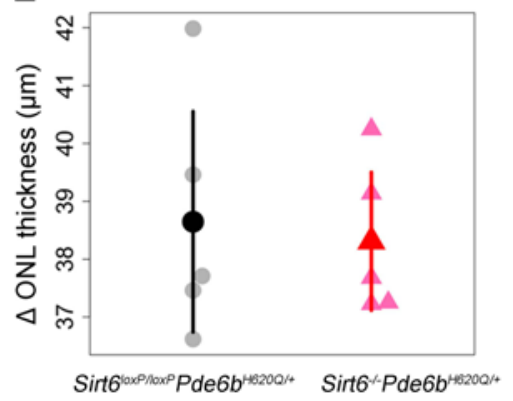

F

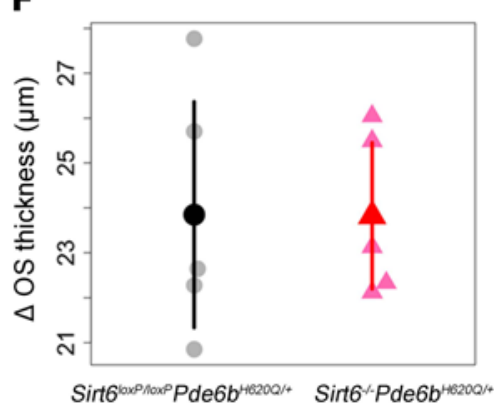

Figure 4. Sirt6 deficiency in WT background produces no phenotypic changes in functionality or morphology of photoreceptors. (A-C) ERG mixed,

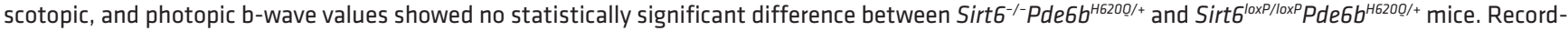
ings were taken 3 months after tamoxifen injection. Gray dots represent values from individual Sirt $\sigma^{10 \times P / l o x P} P d e 6 b^{H 6200 /+}$ mice, while the black dot represents the mean. Similarly, light red triangles represent values from individual Sirt6 ${ }^{-/-P d e 6 b^{H 6200 /+}}$ mice, and the red triangle, the mean. Error bars show SD; $n=5$ for both groups. (D-F) H\&E-stained retinal sections were collected from both groups at 4 months after tamoxifen injection. No differences in ONL or OS thickness were observed. Layer widths were quantified, and results confirmed histological findings. Graphical representations are as those described in A-C. $n=5$ for both groups. Red vertical bars represent ONL; black bars represent IS/OS. Scale bar: $50 \mu \mathrm{m}$.

diminished over time, with no significant difference by 8 weeks. A similar pattern was observed for the mixed rod-cone response (Figure 1B, top), although a statistically significant difference between the groups remained even at 8 weeks. Notably, for the cone response, the b-wave amplitude showed no statistically significant difference between $\operatorname{Sirt}^{-/}{ }^{-} \mathrm{P} d e 6 b^{\mathrm{H} 620 \mathrm{Q} / \mathrm{H} 620 \mathrm{Q}}$ and $\operatorname{Sirt} 6^{\text {loxP/loxP }}$ $P d e 6 b^{\mathrm{H} 620 \mathrm{Q} / \mathrm{H} 620 \mathrm{Q}}$ mice at 4 weeks, but differences became statistically significant at the 6- and 8-week time points (Figure 1B, middle), as would be expected in RP. In general, rods are the primary targets of RP-related degeneration, whereas cones are unaffected until later stages in the disease, after most of the rods have died. Consistent with the later onset of cone dysfunction in RP, a measurable difference in the ERG data acquired from cones in $\mathrm{Sirt6}^{-/-} \mathrm{Pde} 6 b^{\mathrm{H} 620 \mathrm{Q} / \mathrm{H} 620 \mathrm{Q}}$

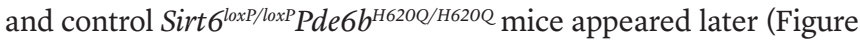
$1 \mathrm{~B}$, middle), when the rods had deteriorated. These data suggest that Sirt6 knockout preserves the electrophysiological function of rods and cones and slows the rate of functional loss.

Morphological rescue of retina layers in Sirt6 ${ }^{-/} P d e 6 b^{H 6200 / H 6200}$ mice. To observe retinal morphology at 3, 4, 6, and 8 weeks, we harvested retinae from experimental and control mice. Some were stained with H\&E (Figure 2A), while others were observed under transmission electron microscopy (TEM) (Figure 2, B and C). For the histology sections, the thickness of the inner segment (IS)/OS layers and the number of nuclei in the ONL were quantified (Figure 2, D and E). The IS/OS of Sirt6 $6^{-/} P d e 6 b^{H 620 Q / H 620 Q}$ mice were more robust in thickness and the ONL number of nuclei was denser compared with $\operatorname{Sirt} 6^{\text {loxP/loxP } P d e 6} b^{\mathrm{H} 620 \mathrm{Q} / \mathrm{H} 620 \mathrm{Q}}$ mice at all but the last time point (Figure 2, A, D, and E). Specifically, both the IS/OS layer and ONL were viable until week 6 in the treated group, whereas they were indiscernible by that time in the untreated group. However, at 8 weeks, there was no statistical difference remaining, and only a few nuclei in the ONL were detected in each group. Furthermore, TEM (Figure 2, B and C) allowed for a minute inspection of the mitochondria and OS for comparison between groups. It was found that the treated group had significantly healthier mitochondria, as indicated by a greater number of cristae, as well as longer OS, with more concentrated disc numbers. While these differences decreased over time, the Sirt6 ${ }^{-1} \mathrm{Pde} 6 b^{\mathrm{H} 620 \mathrm{Q} / \mathrm{H} 620 \mathrm{Q}}$ mice still appeared to have healthier overall mitochondria and OS morphologies, until 8 weeks.

Sirt6 deficiency promotes photoreceptor survival and preserves cellular OS. The OS layer is composed of both rod and cone OS. To distinguish between each cell type, we immunostained retinal sections obtained at 3, 4, and 6 weeks with antibodies directed against rhodopsin to identify rods (red) and opsin for short-wavelength cones (blue) (Figure 3, A-D). We found rod and cone OS were thicker in the Sirt6-deficient mice compared with controls at every time point. Cones were still detectable at week 6 in the Sirt6 ${ }^{-/} P d e 6 b^{H 620 Q / H 620 Q}$ mice, whereas only a few rods and cones with abnormal morphology remained in the Sirt $6^{\text {loxP/loxP } P d e 6} b^{H 620 Q / H 620 Q}$ OS layer. Additional- 


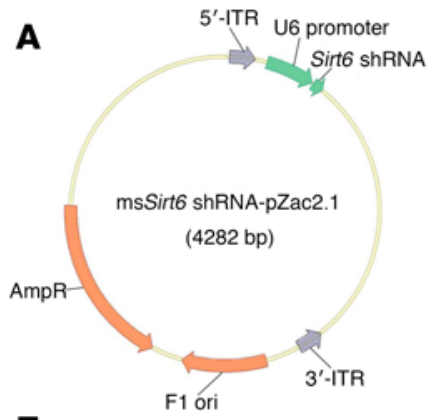

E

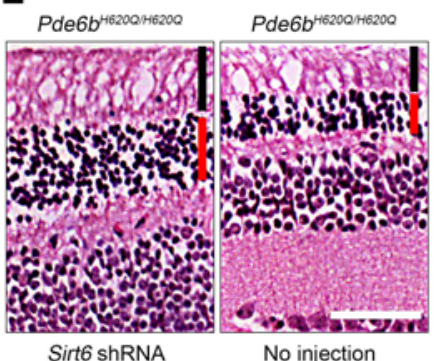

B

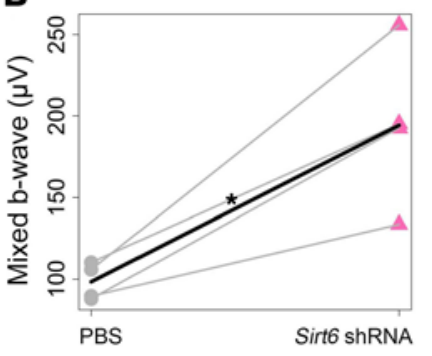

$\mathbf{F}$

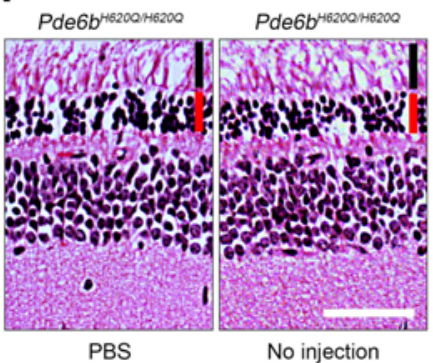

C

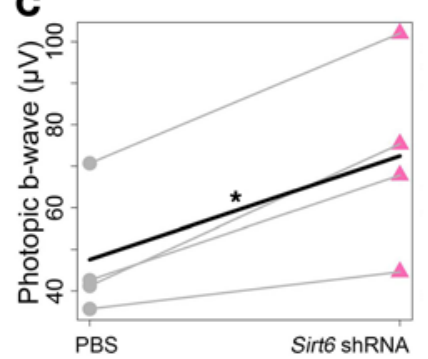

G

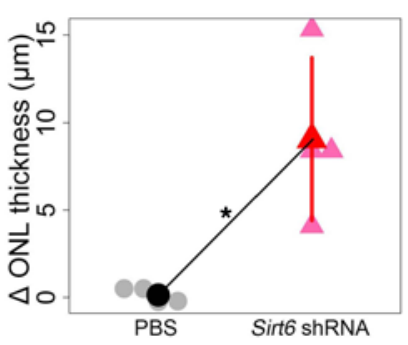

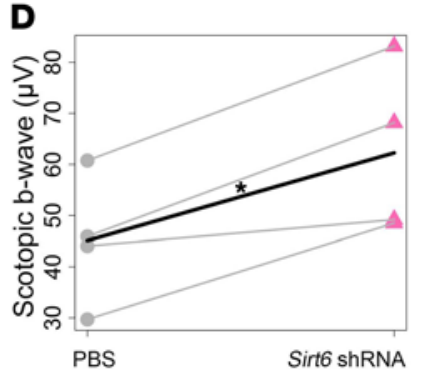

H

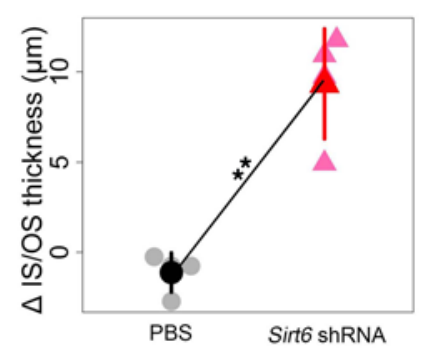

Figure 5. Gene therapy improves function and survival of photoreceptors in a preclinical RP model. (A) Schematic representation of the AAV2/8(Y733F)-Sirt6_ shRNA pZac2.1 vector with the U6 promoter driving expression of the Sirt6_shRNA. Arrows indicate the direction of transcription. 5'- and 3'-ITR, inverted terminal repeats of AAV; AmpR, ampicillin resistance gene; F1 ori, origin of replication. (B-D) Mixed, photopic, and scotopic ERG b-wave recordings ( $\mu \mathrm{V})$ showed a statistically significant difference in the AAV2/8(Y733F)-Sirt6_shRNA-injected eye compared with the PBS-injected eye at 4 weeks after injection. Gray dots represent PBS-injected eyes, while red triangles represent Sirt6_shRNA vector-injected eyes. Gray lines connect eyes from the same mouse; black lines indicate differences in means between PBS-injected and vector-injected eyes. Paired $t$ tests were used to compare groups. Mixed: $P=0.02$; photopic: $P=0.02$; scotopic: $P=0.02$. $n=4$ for all groups. (E-H) H\&E-stained retinal sections were taken from a 4-week-old Pde66 ${ }^{H 6200 / H 6200}$ mouse that was injected dorsally with Sirt6_shRNA in the right eye (E, left). The ventral side of the right eye was not injected but was used as a control (E, right). In the left eye, PBS was injected dorsally (F, left) while the ventral side served as a control (F, right). The dorsal side of the AAV-injected right eye shows retinal rescue (C), while control (PBS-injected) and untreated sites on the ventral side of each eye show continued degeneration $(\mathbf{H})$. Gray dots represent values from individual PBS-injected retinae, while the black dot represents mean thickness. Light red triangles represent values from individual Sirt6_shRNA-injected retinae, and the red triangle, the mean. ONL: $P=0.03 ;$ IS/OS: $P=0.004$. $n=4$ for all groups. Red vertical bar represents ONL; black bar represents IS/OS. Scale bars: $50 \mu \mathrm{m} .{ }^{*} P<0.05$ and ${ }^{* *} P<0.01$.

ly, the OS in the Sirt6 ${ }^{-1}$ Pde $6 b^{H 620 Q / H 620 Q}$ mice were longer than those in the control mice at every time point. This suggests not only that photoreceptor death is slowed in the treated mice, but also that cell morphology is able to resist deterioration for a longer period.

Although the gene responsible for RP in the Pde6b model is expressed exclusively in rods, cone cell death characterizes the late disease stages and causes blindness. Anti-cone arrestin staining (green) identified cone cells, and DAPI staining (blue) identified retinal nuclei (Figure 3, E and F). Cone cell density was higher at every time point in treated (Sirt6 ${ }^{-/}$Pde 6 $b^{H 6200 / H 620 Q}$ ) compared with untreated mice (Figure $3 \mathrm{~F}$ ). In untreated mice, few cones could be detected at 12 weeks, whereas a thin layer remained visible in Sirt6 ${ }^{-1} P d e 6 b^{H 620 Q / H 620 Q}$ mice. In both groups, the greatest decrease in cone cell density occurred between 4 and 6 weeks, which is congruent with the rate of degeneration typical of this mouse model.

Sirt6 deficiency in WT background produces no phenotypic changes in functionality or morphology of photoreceptors. Sirt $6^{-1}$

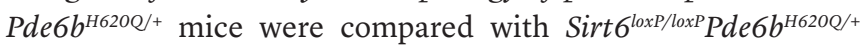
mice at 3 months following tamoxifen injection. ERG recordings of mixed, scotopic, and photopic b-waves were obtained (Figure $4, \mathrm{~A}-\mathrm{C}$ ). No statistical difference between the groups was observed for any of the light adaptation conditions. Retinal sections from each group were harvested at 4 months after tamoxifen injection and were subjected to $\mathrm{H} \& \mathrm{E}$ staining. No difference in OS or ONL thickness was observed (Figure 4, D-F). These results suggest that the Sirt6 deficiency has neither a beneficial nor deleterious effect in the WT background.

Gene therapy-mediated functional and morphological photoreceptor rescue in a preclinical RP model. To translate tamoxifeninduced Sirt 6 ablation into the format of a potential interventional trial, we employed a Sirt6_shRNA somatic gene therapy strategy. An AAV2/8 Sirt6_shRNA vector was transduced into the dorsal retina of the right eye in $P d e 6 b^{\mathrm{H} 620 \mathrm{O} / \mathrm{H} 620 \mathrm{O}}$ mice (Figure 5A). The dorsal retina of the left eye was injected with PBS, whereas the ventral side of all eyes was left untreated. Mixed, photopic, and scotopic ERG b-wave values were recorded and averaged at 4 weeks of age (Figure 5, B-D). The eyes injected with the Sirt6_shRNA vector showed significantly higher $b$-wave values. $\mathrm{H} \& \mathrm{E}$ staining of retinal sections from each eye 4 weeks after injection revealed a measurable increase in the photoreceptor density of vector-injected eyes compared with PBS-injected or untreated eyes (Figure 5, E-H). Notably, even within the same eye, only the dorsal side of right eyes showed increased photoreceptor density, whereas the untreated ventral side of the same eye showed degeneration.

Upregulation of proteins involved in glycolysis in Sirt6-deficient retinae. Because of the critical role of Sirt6 in regulating metabolism, we expected there to be a metabolic basis to the observed photoreceptor rescue (Figures 1-5). Thus, we assessed changes in the flux of the downstream targets of Sirt6 through glycolysis and 
A

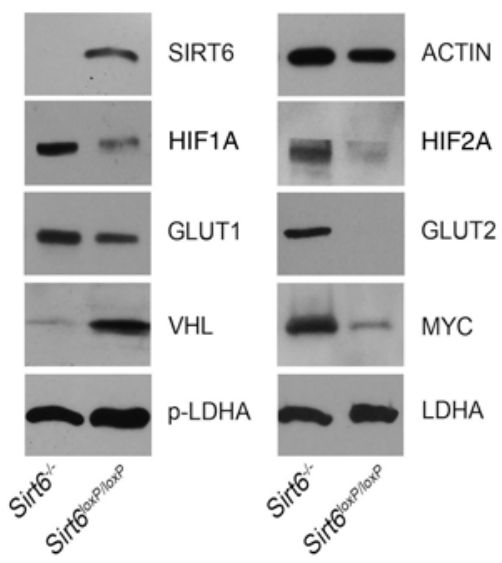

B

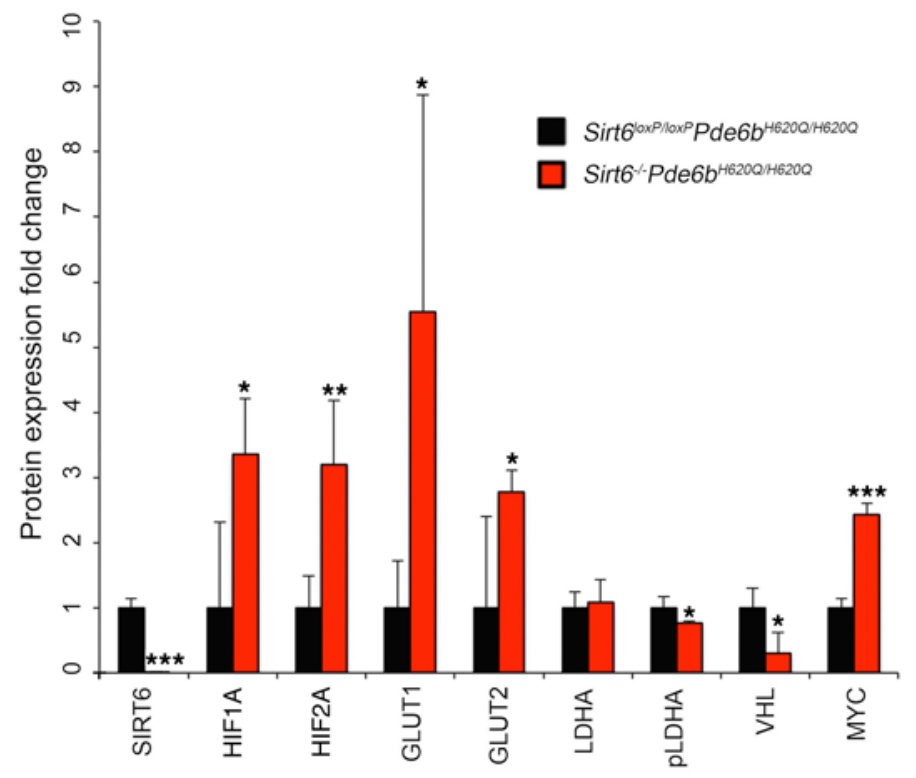

Figure 6. Sirt6 deficiency upregulates levels of glycolytic metabolism intermediates. (A) Immunoblotting for regulators of glycolytic metabolism in the

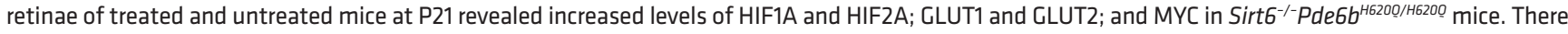
was a corresponding decrease in VHL protein and p-LDHA. No difference was detected for LDHA between the groups. $\beta$-Actin (ACTIN) was used as a loading

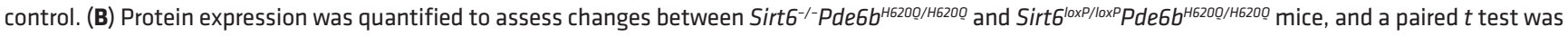
used to determine statistical significance. HIF1A, HIF2A, GLUT1, GLUT2, and MYC were found to be increased in the Sirt6 ${ }^{-/-P d e 6 b^{H 6200 / H 6200}}$ mice compared with controls. SIRT6, VHL, and p-LDHA were found to be decreased in the Sirt6 $/-P d e 6 b^{H 6200 / 46200}$ group. No difference between groups was found for LDHA. SIRT6 $P<0.001$, HIF1A $P=0.02$, HIF2A $P=0.01$, GLUT1 $P=0.04$, GLUT2 $P=0.048$, LDHA $P=0.68$, p-LDHA $P=0.04$, VHL $P=0.02$, MYC $P<0.001$. $n=4$ for both groups. ${ }^{*} P<0.05$, ${ }^{*} P<0.01$, and ${ }^{* *} P<0.001$.

anabolic pathways. Retinal extracts from Sirt6-deficient retinae were collected, and levels of the proteins involved in glycolysis were measured by immunoblotting (Figure 6; see complete unedited blots in the supplemental material.). These included HIF1A and HIF12A; glucose transporters GLUT1 and GLUT2; and MYC - all of which were increased compared with the levels observed in extracts from control retinae (Figure 6). Upregulation of these downstream factors suggests increased levels of anabolism in Sirt6 ${ }^{-}$Pde $6 b^{H 620 Q / H 620 Q}$ retinae. Phosphorylated lactate dehydrogenase A (p-LDHA) and von Hippel-Lindau (VHL) protein were downregulated in Sirt6 ${ }^{-}-\mathrm{Pde} 6 b^{\mathrm{H} 620 \mathrm{Q} / \mathrm{H} 620 \mathrm{O}}$ mice, while LDHA levels showed no difference between groups. LDHA is an enzyme that converts pyruvate into lactate, and some studies suggested that phosphorylation of LDHA increases its activity and induces the Warburg effect $(49,50)$, although this does not appear to be the case in Sirt6-deficient retina. VHL regulates protein degradation and downregulates HIF2A, which is reflected in our data by the decreased levels of VHL and the increased levels of HIF2A.

Analysis of downstream metabolites upregulated by Sirt6 deficiency. To directly determine the effects of Sirt6 deficiency on the rate of glycolysis and the TCA cycle, we used gas chromatographymass spectrometry (GC-MS) analysis to assess flux through key metabolic intermediates (Figure 7A) at the onset of degeneration as measured by the fractional abundance of each intermediate. Forty-five minutes after i.p. injection of ${ }^{13} \mathrm{C}$-labeled glucose, retinae were harvested, and the distribution of metabolite isotope isomers (isotopomers) was analyzed to measure the enrichment of each intermediate by determining the ratio of labeled ions to total ion intensity at 3 and 4 weeks of age (Figure 7B). ${ }^{13} \mathrm{C}$-labeled glucose is transformed into pyruvate and lactate through glycolysis, and the pyruvate enters into the mitochondria and is incorporated into the TCA cycle as metabolic intermediates and amino acids. Retinae in Sirt6 $6^{-1}$ Pde $6 b^{\mathrm{H} 620 \mathrm{Q} / \mathrm{H} 620 \mathrm{O}}$ mice showed much higher ${ }^{13} \mathrm{C}$-incorporated intermediates in both glycolysis and the TCA cycle compared with controls at both time points. However, at 4 weeks the differences were diminished and in some cases were not statistically significant, suggesting that the degeneration may have advanced beyond what Sirt6 deficiency could compensate for. The relative abundance of metabolic intermediates in the control and experimental mice, as measured by the total number of carbons labeled in each metabolite, was significantly increased for glycolytic intermediates, more so than for TCA cycle intermediates; this indicated that Sirt6 deficiency, while increasing overall metabolism, exerts its greatest effects on glycolysis (Figure 7, C and D). This shift may be beneficial for enhancing anabolic processes in the Sirt6 $6^{--}$ $P d e 6 b^{\mathrm{H} 620 \mathrm{O} / \mathrm{H} 620 \mathrm{O}}$ retinae. To determine whether the absolute abundance of the metabolites was also increased, we performed liquid chromatography-mass spectrometry (LC-MS) when mice were 3 weeks of age to analyze intermediates involved in the major glucose metabolism pathways, detecting 126 in total (Supplemental Figure 2-4). More than 100 of the metabolites were upregulated in the Sirt6-deficient mice compared with controls, although the difference was significant only for some of them. All metabolites with a significant difference $(P<0.05)$ are shown (Figure $7 \mathrm{E})$ and were upregulated in the Sirt6- ${ }^{-}$Pde $6 b^{H 620 Q / H 620 O}$ mice at 3 weeks. These experiments were repeated at 4 weeks, and the majority of metab- 
A
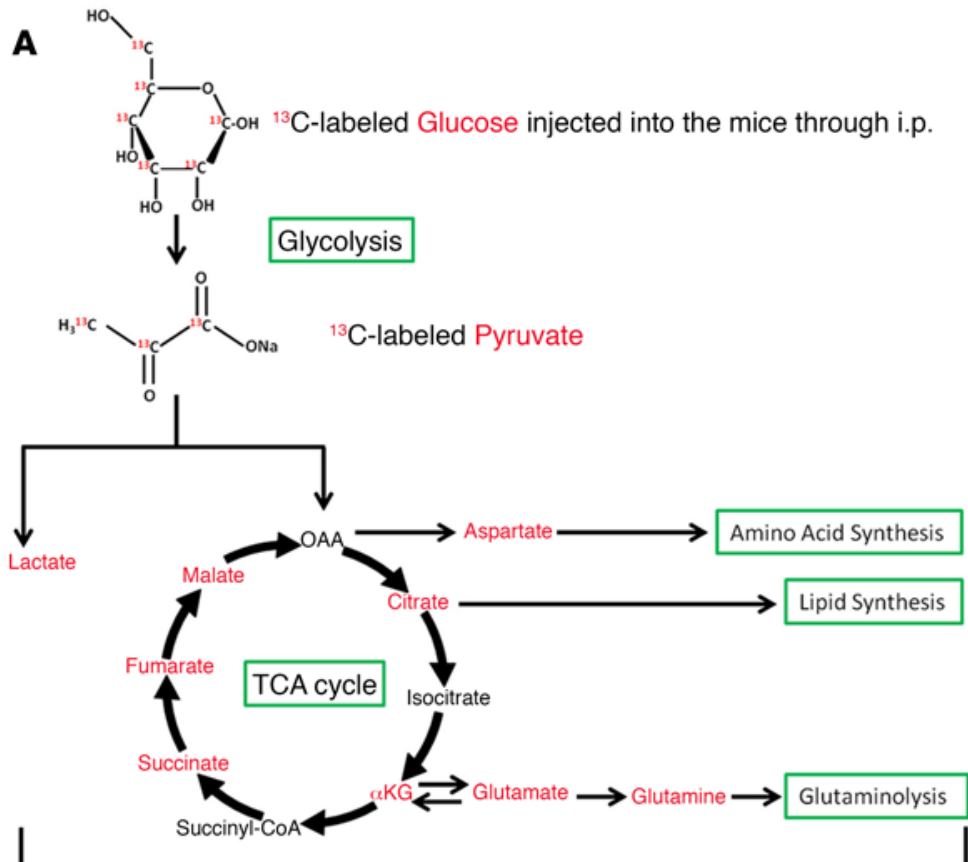

Isolate informative metabolites from retinas

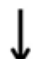

Analyze for abundance and ${ }^{13} \mathrm{C}$ enrichment

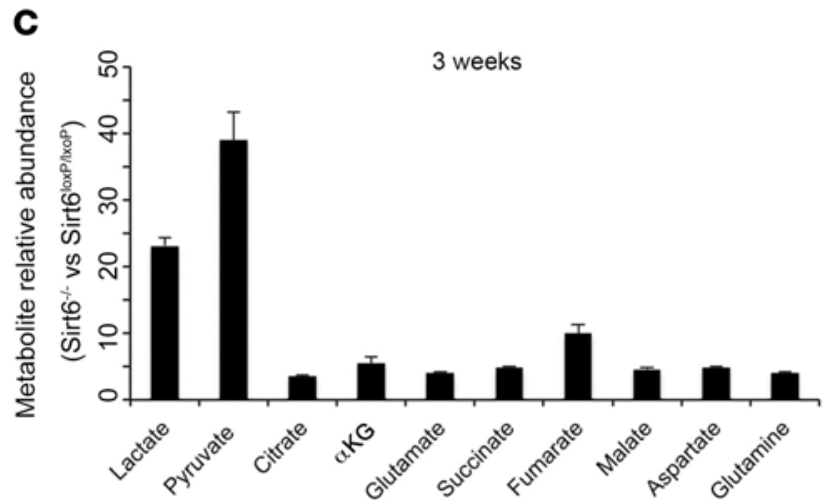

B

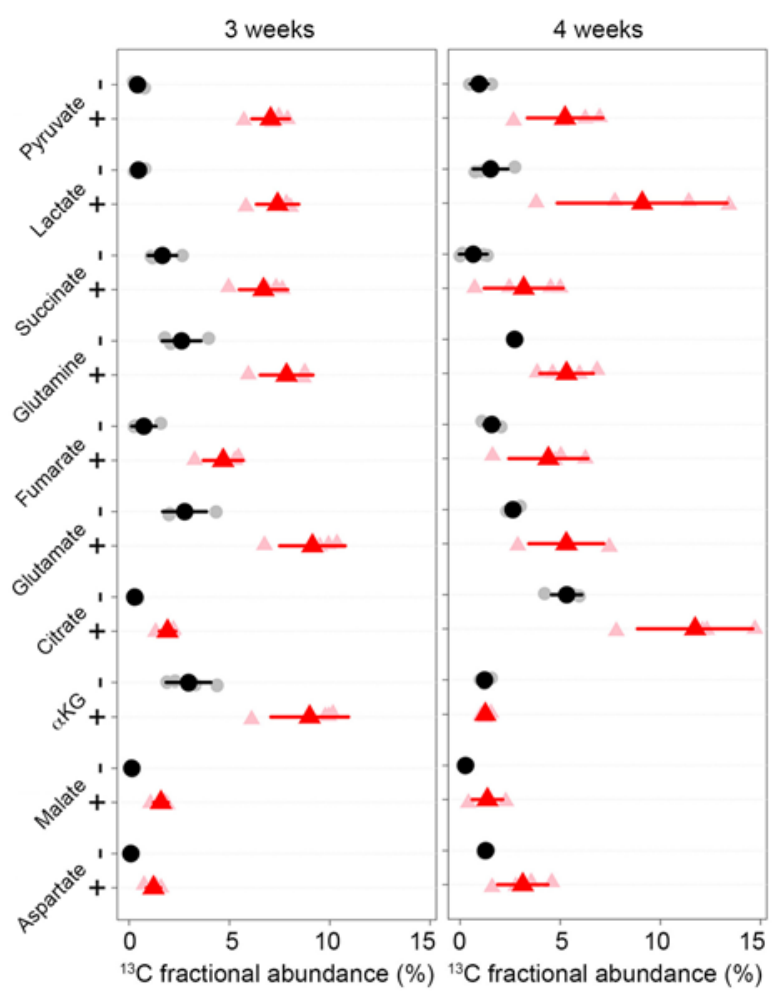

D

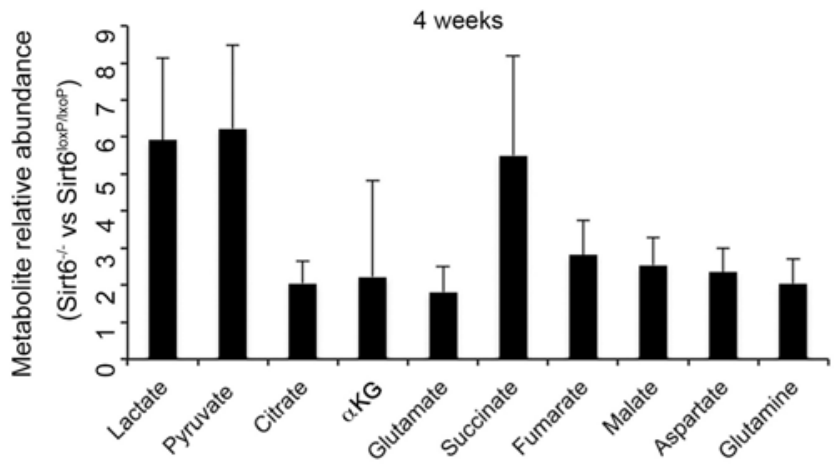

E

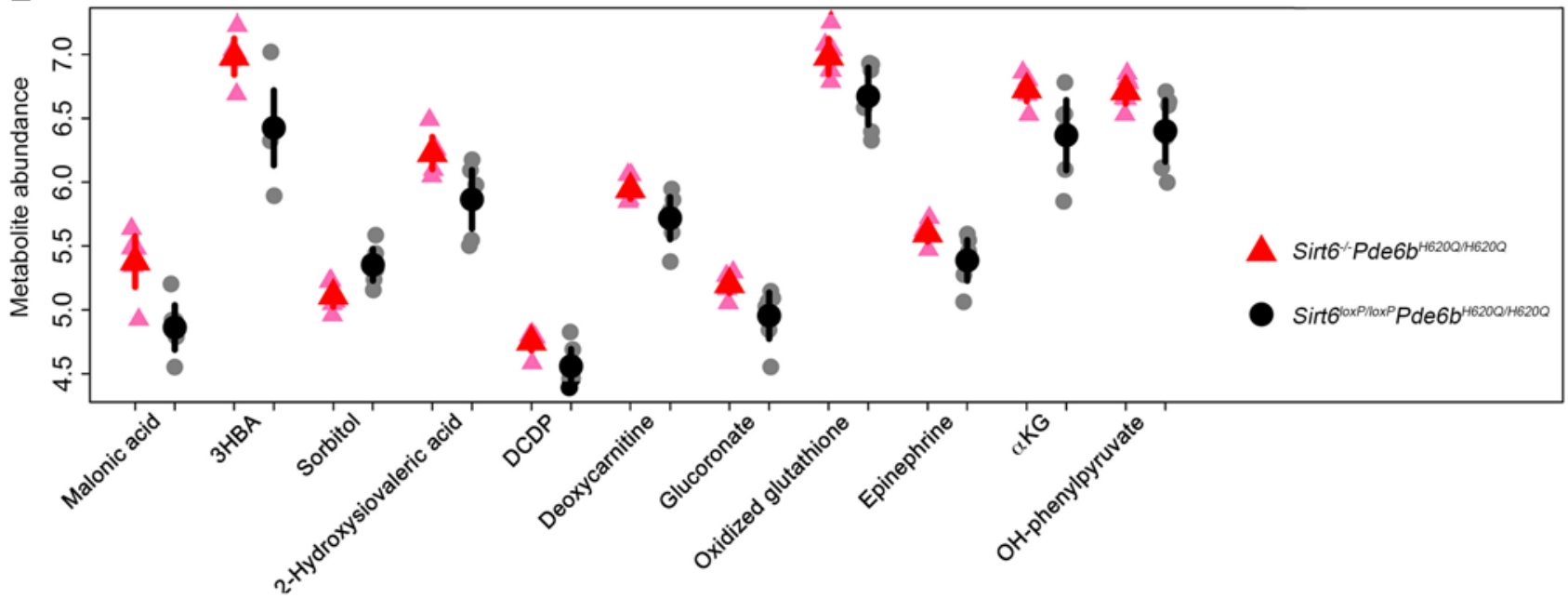


Figure 7. Sirt6 knockout accelerates the flow of carbons from glucose to downstream metabolites. (A) Schematic representation of glycolysis and the TCA cycle, highlighting key metabolic intermediates that are compared between groups in B. Red font indicates incorporation of the ${ }^{13} \mathrm{C}$ isotope into the downstream metabolites evaluated by GC-MS. (B) The ratio of ${ }^{13} \mathrm{C}$ enrichment of each metabolite at 3 and 4 weeks is shown. At 3 weeks, Sirt6 ${ }^{-1-P d e 6 b^{H 6200 / 46200}}$ mice have significantly higher levels of ${ }^{13} \mathrm{C}$-labeled metabolites compared with controls. At 4 weeks, the significance remained for only some metabolites: pyruvate, citrate, glutamine, and lactate. Gray dots and light red triangles represent values from individual Sirt6 $6^{10 \times P / 10 \times P} P d e 6 b^{H 6200 / 146200}$ and Sirt6 ${ }^{-1-P d e 6 b^{H 6200 / 46200}}$ mice, respectively. Black dots and red triangles represent the mean. -, Sirt6 ${ }^{-1-P d e 6} b^{H 6200 / H 6200}$ mice; + , Sirt6 $6^{10 \times P / 10 \times P} P d e 6 b^{H 6200 / H 6200}$ mice. Two-tailed $t$ tests were used for analysis. $P<0.001$ for all metabolites at 3 weeks. $P<0.05$ for pyruvate, citrate, glutamine, and lactate at 4 weeks. $P=$ NS for other metabolites. $n=4$ for Sirt6 $6^{-1-P d e 6 b^{H 6200 / H 6200}}$ and Sirt6 ${ }^{10 \times P / 10 \times P} P$ de $6 b^{H 6200 / 46200}$ mice at 3 and 4 weeks. (C and D) Metabolic relative abundance (fold change) of glycolytic and TCA cycle intermediates was assessed at 3 weeks of age. All metabolites were significantly upregulated in the treated group. Similar results were obtained at 4 weeks but with diminished significance. Three weeks, all metabolites: $P<0.001 ; n=4$ for both groups. 4 weeks, lactate: $P=0.009$; pyruvate: $P=0.005$; glutamine: $P=0.02$; malate: $P=0.03$; fumarate: $P=0.04$; aspartate: $P=0.03$; all others: $P>0.05 . n=4$ for both groups. (E) LC-MS (without ${ }^{13} \mathrm{C}$ labeling) revealed 11 significantly increased downstream metabolites in the Sirt6 ${ }^{-1-} P d e 6 b^{H 620 / H 6200}$ retinae at 3 weeks of age. Two-tailed $t$ tests were used for analysis. $P<0.05$ for all metabolites; $n=6$ per group.

olites were still increased in Sirt6-deficient retinae, although the significance diminished over time (Supplemental Figures 2-4). Of the 11 metabolites identified at week 3 as having the most significant difference between experimental and control groups, only one maintained significance at week 4: oxidized glutathione (Supplemental Figure 2, C and D). Overall, these results indicate upregulation of glycolytic metabolism in the Sirt6-deficient retina, although this trend decreased over time.

PFK shRNA viral knockdown exacerbates retinal degeneration in Pde6b ${ }^{\mathrm{H} 620 \mathrm{Q} / \mathrm{H} 620 \mathrm{Q}}$ mice. Phosphofructokinase (PFK) is a key regulator of glycolysis and serves as the rate-limiting glycolytic enzyme in metabolism (51). This enzyme has several isoforms (52), including PFK-M, -L, and -P. To isolate the effects of glycolysis on retinal degeneration from the effects of Sirt6 deficiency, we injected a lentivirus expressing shRNA for one of the 3 isoforms into $P d e 6 b^{H 620 Q / H 620 Q}$ mice. Additionally, a lentivirus carrying shRNA for all 3 isoforms (PFK-M+L+P) was injected into a fourth group of mice. ERG mixed, scotopic, and photopic b-wave values acquired at 4 weeks after injection revealed a significant decrease in scotopic b-wave amplitudes in mice injected with shRNA for PFK-M+L+P (Figure 8, A-C). A considerable but not statistically significant decrease was observed in mixed and photopic b-wave values. H\&E-stained retinal sections confirmed these findings (Figure 8, D-F). A significant decrease in ONL and OS layer thicknesses was detected in the PFK-M+L+P shRNA group. As PFK is directly responsible for activating glycolysis, these findings, particularly the histological results, support our hypothesis that metabolic imbalances may underlie or at least exacerbate degeneration.

\section{Discussion}

Inherited photoreceptor degenerative conditions are pervasive and devastating, often leading to loss of the ability to conduct activities of daily living (1,53-55). RP, a debilitating neurodegenerative disorder, is associated with at least 64 genes encoding mostly rod cell-specific proteins that lead to cell death when improperly formed (56-58). There is currently no cure available, and although gene therapy interventions recently reached clinical trials, the heterogeneity of gene deficits that cause hereditary neurodegenerative conditions is a fundamental limitation of these studies $(26,27,29,59-64)$; this is because the strategy involves a monotherapy, which cannot be used to treat disorders caused by mutations in more than one gene. Thus, a non-gene-specific rescue strategy $(11,12)$ to increase biosynthetic fuel and survival by reprogramming of glycolysis is highly desirable.
Photoreceptors are among the most metabolically active cells in the body (15-19), converting $80 \%-96 \%$ of glucose into lactic acid via aerobic glycolysis (65). RP-induced rod cell death is theorized to arise from defective metabolic processes in diseased cells, among other causes (22). We therefore hypothesized that degeneration can be rescued by tipping the cell's metabolic balance toward anabolism through inhibition of Sirt6, a transcriptional repressor of glycolytic flux. This is based on the theory that aerobic respiration typically leads to catabolic processes, while anaerobic metabolism caters to anabolism $(66,67)$.

SIRT6 is already known as a regulator of glycolysis (40), but it has yet to be studied as a therapeutic target for reprogramming metabolism in neurodegeneration. Studies show that SIRT6 causes glucose to be preferentially shuttled into aerobic respiration, and in its absence, anaerobic metabolic pathways, glycolysis in particular, are prioritized instead. Here, we ablated Sirt6 in a preclinical model of RP to assess the role metabolism plays in retinal degeneration. We hypothesized that cells could be preserved by upregulating anabolism, which could be achieved by prioritization of glycolysis caused by Sirt6 deficiency.

In the present study, electrophysiological and anatomic rescue of the Pde $6 b^{\mathrm{H} 620 \mathrm{O} / \mathrm{H} 620 \mathrm{O}}$ preclinical model was achieved early on in the disease with both germline and AAV2/8 Sirt6_shRNA somat-

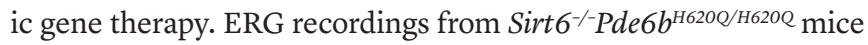
showed higher $b$-wave amplitudes, thicker OS/ONL, and greater rod and cone nuclear density compared with controls. Because the Pde $6 b^{\mathrm{H} 620 \mathrm{Q} / \mathrm{H} 620 \mathrm{O}}$ mutation is hypomorphic, the PDE6 protein remains partially active, providing an explanation for the measurable ERG recordings obtained in the control mice before degeneration overwhelmed the functionality of the photoreceptors (10). Loss of Sirt6 increased levels of HIF1A, HIF2A, GLUT1, GLUT2, and MYC, which are key drivers of biosynthesis. Increased levels of HIF1A and GLUT1 have previously been associated with photoreceptor survival $(11,68)$. In one study, injection of insulin in 4 unique RP mouse models was shown to delay degeneration of cones, and increased levels of GLUT1 and HIF1A were detected (11). In another study involving tuberous sclerosis complex 2 (TSC2) ablation, the mTOR pathway was enhanced, leading to upregulation of GLUT1, which was hypothesized to protect cones from degeneration (12). Additionally, GLUT1 was shown to play an important role in protecting rods via rod-derived cone viability factor (RdCVF) (69). Sirt6 deficiency allowed maximal activity of enzymes involved in both glutaminolysis, driven by MYC (70), and enhanced retinal glycolytic flux. Increasing levels of HIF1A and MYC were shown to upregulate LDHA in cancer cells (50), which was not observed 
A

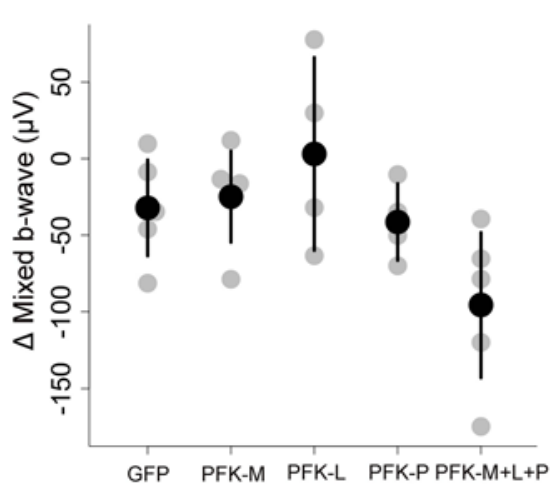

D

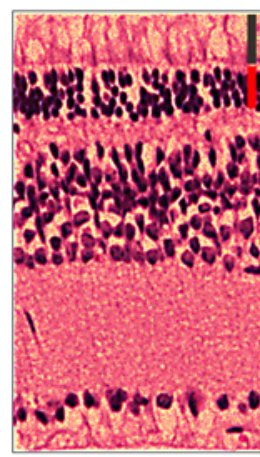

No injection
B

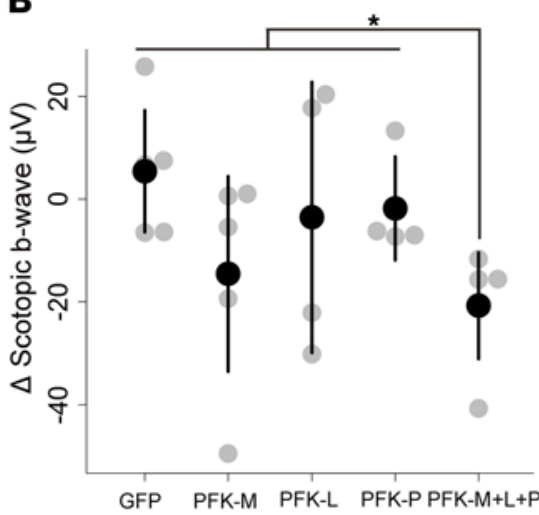

$\mathbf{E}$

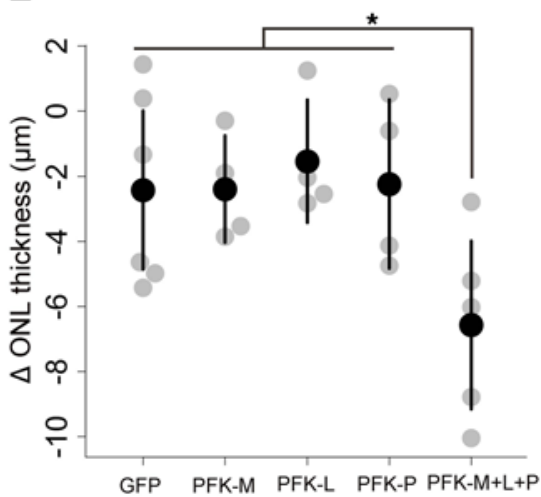

C

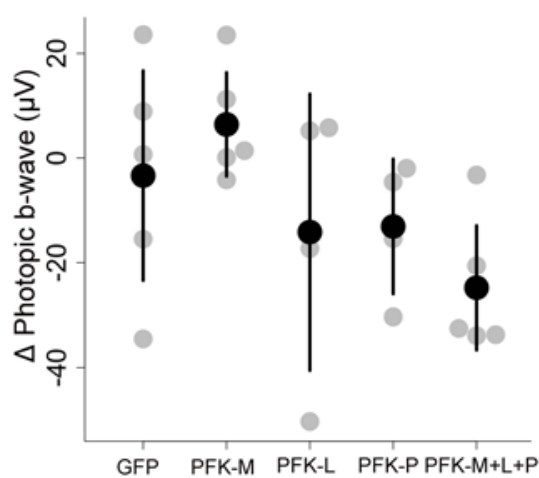

$\mathbf{F}$

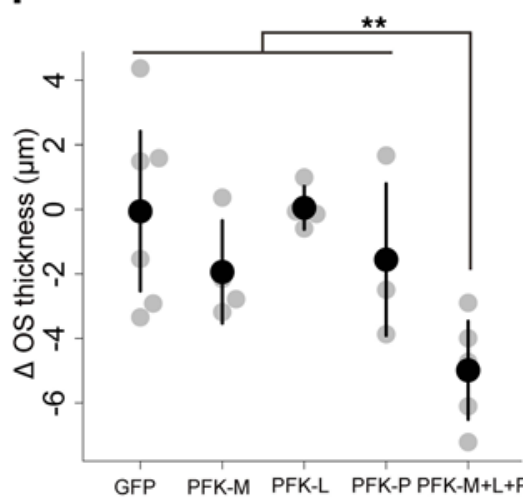

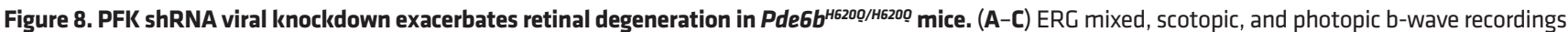

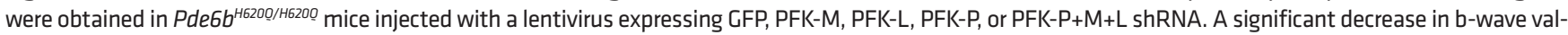
ues under scotopic conditions was detected following PFK-P+M+L shRNA introduction, and a notable but nonsignificant difference was detected under mixed and photopic conditions. Gray dots represent values from individual mice, while the black dot represents the mean. Pairwise comparisons against GFP were conducted for each viral injection: Scotopic - PFK-M: $P=0.12$; PFK-L: $P=0.57$; PFK-P: $P=0.38$; PFK-P+M+L: $P=0.01$. Mixed - PFK-M: $P=0.74$; PFK-L: $P=0.37$; PFK-P: $P=0.66$; PFK-P+M+L: $P=0.06$. Photopic - PFK-M: $P=0.42$; PFK-L: $P=0.53$; PFK-P: $P=0.44$; PFK-P+M+L: $P=0.11$. $n=5$ for all groups except PFK-L, where $n=4$. (D-F) Mice were injected dorsally with the lentivirus, while the ventral side was left untreated. An observable difference was detected between the injected and noninjected sides in mice exposed to the lentivirus expressing PFK-P+M+L shRNA. The difference in ONL and OS thickness was quantified and found to be statistically significant for the PFK-P+M+L injection group compared with GFP controls. Graphical representations were rendered as described above and showed that only the PFK-P+M+L combination led to a statistically significant decrease in each layer. Red bar represents ONL; black bar represents $0 S$. Scale bar: $20 \mu \mathrm{m}$. Pairwise comparisons against GFP were conducted for each viral injection: ONL - PFK-M: $P=0.98 ; P F K-L: P=0.58 ; P F K-P: P=0.92 ; P F K-P+M+L: P=0.04$. OS $-P F K-M$ : $P=0.24$; PFK-L: $P=0.93$; PFK-P: $P=0.41$; PFK-P+M+L: $P=0.01 . n=5$ for all groups except PFK-L, where $n=4$. ${ }^{*} P<0.05$ and ${ }^{* *} P<0.01$.

in the Sirt6-deficient retina. The discrepancy between our data and prior studies could be explained by a negative feedback mechanism, in which Sirt6 knockout increases anabolic and glycolytic metabolites, including lactate (Figure 7), and the resulting high levels of lactate repress LDHA activity and phosphorylation. Furthermore, the ${ }^{13} \mathrm{C}$-labeled isotopomer data reliably confirmed that glycolytic flux was preferentially upregulated $(71,72)$.

Overall, the metabolome shifted to accumulate biosynthetic intermediates in the OS of rods lacking Sirt6, including intermediates in multiple metabolic pathways such as glycolysis, the TCA cycle, and glutaminolysis (Figure 7, A-D). These changes are consistent with upregulated anabolism, which, in turn, would be expected to improve retinal function and counter cell death. Additionally, the expression of the metabolites was enhanced in Sirt6 ${ }^{-/}-P d e 6 b^{H 620 Q / H 620 Q}$ mice as assessed by LC-MS (Figure 7E and Supplemental Figures 2-4). After statistical analysis, we confirmed that glycolytic metabolism was enhanced, as indicated by increased flux from glucose into pyruvate and lactate. The intermediates of the TCA cycle were also upregulat- ed, but to a lesser extent (Figure 7, C and D). For example, the flux from glucose to $\alpha$-ketoglutarate $(\alpha \mathrm{KG})$ was dramatically increased in the Sirt6-deficient mice compared with controls. Plausibly, Sirt6 deficiency enhanced glycolysis, and the glycolysis-derived pyruvate entered the mitochondria to increase $\alpha \mathrm{KG}$ and other intermediates. Overall, the fold change in lactate and pyruvate, both end points of glycolysis, was significantly higher compared with metabolites of the TCA cycle, suggesting a preference for glycolytic metabolism in the Sirt6-deficient mice.

To confirm that glucose metabolism influences retinal degeneration, we knocked down all PFK isoforms in the Pde6 $b^{\mathrm{H} 620 \mathrm{O} / \mathrm{H} 620 \mathrm{Q}}$ background, as this enzyme regulates the rate-limiting step in glycolysis and its intermediates feed into the TCA cycle. Knockdown significantly decreased ERG scotopic b-wave amplitudes and cell layer thickness (Figure 8), suggesting that glycolytic aberrations do affect retinal degeneration. However, there are multiple downstream enzymes and alternative pathways that could be responsible for these results, and further experiments will be needed to explore other possibilities. 
While the reduced rate of disease progression is encouraging, a limitation of our approach is that Sirt6 ablation counteracted, but could not completely halt, cell death. Indeed, despite decades of research, no therapy has been discovered that can entirely halt disease progression, although several strategies have been able to slow the rate of cell death. Why Sirt6 deficiency confers early-stage but not long-term disease amelioration remains unclear. The primary cell death drivers, $\mathrm{Ca}^{2+}$ and cGMP toxicity arising from PDE6 dysfunction, were not reversed by Sirt6 inhibition. Continued cone cell death may be due to loss of RdCVF after death of rods (73-77) or because of a reduction in the antioxidant transcription factor $\operatorname{NRF} 2(78,79)$. Achieving long-term efficacy is a universal limitation for most gene therapy interventions. In recent clinical trials of retinal gene therapy in Leber congenital amaurosis, some research subjects showed functional improvements (25-32). However, several follow-up studies clearly showed that photoreceptor loss had not been halted or even slowed (59, 80-82). Another limitation of our data is that they may only be applicable to RP associated with the Pde6 mutation. Future studies should be undertaken to explore whether metabolism plays a similar role in other RP disease origins.

Sirt6_shRNA gene therapy achieved efficiency similar to Opsin::Pde6b gene therapy, where a viral transgene expressing WT $P d e 6 b$ was able to induce partial functional and localized morphological rescue of photoreceptors (10). A future strategy would be to apply a bipartite gene therapy vector to treat a patient's specific mutation(s) while simultaneously reprogramming anabolism. A combinatorial therapy approach could potentially prevent future damage. Another alternative may be to combine downregulation of Sirt6 with upregulation of the mTORC1 pathway, as described by Venkatesh et al. (12). This would involve inhibition of Tsc1 in the cones of Sirt6 $^{-1-} P d e 6 b^{H 620 Q / H 620 Q}$ mice. Upregulation of anabolism in rods combined with simultaneous upregulation of mTORC1 in cones could, perhaps, have additive effects on photoreceptor survival compared with the use of each strategy in isolation. Targeting other key regulators to reprogram metabolism may also prove fruitful in developing treatments for RP. In general, the metabolic underpinning of RP is not well understood, although mouse models have been used to explore the role metabolism plays in degeneration. Extensive future studies should be conducted to determine whether our results are translatable to other genetic backgrounds of RP and to ascertain the role that the downstream targets of Sirt6 play in neurodegeneration.

Our findings support recent evidence showing that upregulation of glycolysis in cone photoreceptors drives their survival and is crucial to photoreceptor health (69). They also align with reports that increased levels of HIF1A, HIF2A, GLUT1, GLUT2, MYC, and the other glycolytic enzymes are associated with photoreceptor survival (68). Together, these results provide a foundation that supports a role for reprogramming metabolism to treat neurodegenerations. Neurodegenerative conditions such as Alzheimer disease, Parkinson disease, and glaucoma have also been suggested to arise from metabolic aberrations $(59,83,84)$. Thus, our strategy in reprogramming metabolism by targeting Sirt6 signaling may be translatable to halting other degenerative disorders of the central nervous system as well $(85,86)$.

\section{Methods}

Animals. Three lines of mice were crossed to develop the breeding strains. Sirt $6^{t m 1.1 C x d} / J$ mice (87) were purchased from the Jackson Laboratory; Pde6 $b^{\mathrm{H} 620 \mathrm{Q} / \mathrm{H} 620 \mathrm{Q}}$ mice were rederived via oviduct transfer using European Mouse Mutant Archive (EMMA) morulae (10, 88); and Pde $6 g^{\text {CreERT2 }}$ mice were generated in the Barbara \& Donald Jonas Stem Cell and Regenerative Medicine Laboratory (5, 6, 89-94). All mice were housed in the Columbia University Pathogen-Free Eye Institute Annex Animal Care Services Facility and maintained with a 12-hour light/12-hour dark cycle.

Pde6 $b^{\mathrm{H} 620 \mathrm{Q} / \mathrm{H} 620 \mathrm{Q}}$ mice were crossed with Pde6g $g^{\text {CreERT2 }}$ mice, and their offspring were bred with Sirt $6^{t m 1.1 C x d / J}$ mice. Six generations of backcrosses were required to generate breeding mice. The resulting progeny were homozygous for all alleles of interest (Pde6b, Sirt6, and Pde6g), but some were WT at Pde6g, whereas others possessed the Pde6g CreERT2 mutation. We isolated these two lines for use as breeding strains. Crossing the breeding strains produced the experimental mice, which are homozygous at the Pde6b and Sirt6 loci, and heterozygous at the Pde6g locus.

At P7, half of the experimental mice were given a $100 \mu \mathrm{g} / \mathrm{g} \mathrm{BW}$ injection of tamoxifen (100 mg/ml in ethanol; T5648; Sigma-Aldrich), which was diluted with corn oil to a concentration of $10 \mathrm{mg} / \mathrm{ml}$ and thoroughly mixed at $42^{\circ} \mathrm{C}$. One injection was administered on P7, P8, and P9. The other half of the experimental mice were injected with ethanol (10\% in corn oil) following the same dosage as tamoxifen and served as the control group. There was no discrimination based on the sex of the mice.

Genotyping. Recombination of the Sirt6 allele was accomplished through tamoxifen-induced Sirt6 LoxP removal at P7. To verify Sirt6 recombination in rods, $10-\mu \mathrm{m}$ frozen sections of the retina were collected, and DNA was extracted from the ONL using a 30-gauge needle and surgical microscope (m690; Leica). PCR was completed as previously outlined (89). All other organs were subsequently collected. Three primers were used to target the Sirt6 DNA sequence: forward 5'-GCTAATGGGAACGAGACCAA-3'; internal 5'-ACССАССТСТСТССССТАAA-3'; and reverse 5'-GCGTCCACTTCTCTTTCCTG-3'. The recombination allele of Sirt6 was amplified using the forward and reverse primers to produce the 524-bp fragment.

DNA for genotyping was extracted from mouse tails. Sirt6 mice genotypes were confirmed using the forward and internal primers. This primer set amplifies 390-bp fragments for WT Sirt6 mice and 444-bp fragments for mice with conditional alleles that contain the LoxP insertion site in intron 1 . Genotyping $P d e 6 b^{\mathrm{H} 620 \mathrm{Q} / \mathrm{H} 620 \mathrm{Q}}$ required the following primers: forward 5'-TGCCACGACATCGACCACCCG-3' and reverse 5'-GCCATCCCTGCCTTCCCTTGG-3'. This set amplifies a 598-bp fragment, which was sequenced to confirm the presence of the H620Q point mutation. Pde $6 g^{\text {CreERT2 }}$ mice require the following primers: forward 5'-GGTCAGATTCCAGTGTGTGGG-3' and reverse 5'-GTTTAGCTGGCCCAAATGTTG-3'. This primer set produces 514-bp fragments for WT mice and 715-bp fragments for mice with conditional alleles.

Immunoblotting. Retinae were harvested from 3-week-old mice and homogenized in M-PER Mammalian Protein Extraction Reagent (\#78501 Thermo Scientific) supplemented with phosphatase inhibitor cocktail 1 (P2850-5ML; Sigma-Aldrich) and protease inhibitor cocktail (P8340-1ML; Sigma-Aldrich), using a previously published method (5, 
6); and protein concentrations were measured using the bicinchoninic acid (BCA) protein assay (Thermo Scientific). Two eyes from one mouse were run together as one sample. SDS-PAGE (4\%-15\%; Bio-Rad) was used to separate proteins, which were subsequently transferred to nitrocellulose (Bio-Rad). After blocking in 5\% skim milk (902887 MP Biomedicals), membranes were incubated overnight at $4^{\circ} \mathrm{C}$ in the following antibodies: rabbit polyclonal anti-Sirt6 - ChIP Grade (1:500; ab105391; Abcam); rabbit polyclonal anti-VHL - N-terminal (1:100; ab135576; Abcam); mouse monoclonal anti-glucose transporter GLUT1 (1:2,000; ab40084; Abcam); mouse monoclonal anti-glucose transporter GLUT2 (1:2,000; ab104622; Abcam); mouse monoclonal HIF1A (1:500; MBS143031; Novus Biologicals); rabbit polyclonal HIF2A/EPAS1 antibody (1:1,000; NB100-122; Novus Biologicals); mouse monoclonal (9E10) to c-Myc (HRP) (1:1,000; ab62928; Abcam); rabbit polyclonal LDHA antibody (1:500; \#2012; Cell Signaling Technology); p-LDHA (Tyr10; 1:1,000; 8176S; Cell Signaling Technology); and mouse anti- $\beta$-actin (1:1,000; ab125248; Abcam). They were then washed 3 times in $0.5 \%$ PBST ( $500 \mu \mathrm{l}$ Tween-20 in 1,000 $\mathrm{ml} \mathrm{PBS)} \mathrm{and}$ incubated for 1 hour at room temperature in goat anti-rabbit IgG-HRP secondary antibody (1:2,000; sc-2004; Santa Cruz Biotechnology Inc.) or rabbit anti-mouse IgG-HRP antibody (1:2,000; sc-358914; Santa Cruz Biotechnology Inc.). Membrane proteins were revealed by chemiluminescence detection (EMD Millipore) using BioMax film (Kodak).

ONL density and IS/OS length measurement. Mice were euthanized and eyes enucleated according to Columbia University IACUC guidelines following previously described procedures $(5,6)$. The cornea and lens were dissected, and the vitreous was removed, isolating the eyecup. Excalibur Pathology Inc. prepared H\&E and retinal paraffin sections $(5 \mu \mathrm{m})$. To quantify cell numbers and thickness, each section was divided into four $230 \times 230 \mu \mathrm{m}^{2}$ square regions: peripheral temporal; central temporal; central nasal; and peripheral nasal quadrants. ONL density was measured by counting photoreceptor nuclei in each quadrant by determining the number of nuclei located $1 \mathrm{~mm}$ from the optic nerve in each quadrant. This value was then divided by the length of the ONL measured in the 4 quadrants and the thickness of the section. The IS/OS length was determined by measuring the average thickness of the IS/OS layer in the 4 quadrants using Image (NIH).

TEM. Retinae were sectioned, fixed in half-strength Karnovsky fixative, stained with uranyl acetate and lead citrate, embedded in Spurr's medium, cut at $90 \mathrm{~nm}$, collected on grids, and examined by TEM using a Zeiss 190. Images were digitized and viewed in Adobe Photoshop, and slight adjustments were made to the brightness to distinguish mitochondria and OS layers more clearly.

Immunohistochemistry. For frozen sections, eyes were enucleated and placed in $4 \%$ paraformaldehyde for 1 hour at room temperature. After fixation, retinae were dissected from the eyecup, cryoprotected in $30 \%$ glucose overnight at $4^{\circ} \mathrm{C}$, and sectioned vertically at $10 \mu \mathrm{m}$ with a cryostat (Leica). Sections were washed 3 times with PBS ( $\mathrm{pH} 7.4$ ) and incubated overnight at $4^{\circ} \mathrm{C}$ with the following primary antibodies: rabbit anti-cone arrestin (1:5,000, Millipore), mouse anti-rhodopsin (1:500, Santa Cruz Biotechnology Inc.), and rabbit anti-blue opsin (1:200, Millipore) diluted in 5\% Chemiblocker (Life Technologies) and $0.3 \%$ Triton $\mathrm{X}-100$ in PBS. After washing in PBS, the sections were incubated with secondary antibodies conjugated to either Alexa Fluor 555 or Alexa Fluor 488 (1:500, Molecular Probes, Life Technologies) for 1 hour at room temperature. Sections were then washed with PBS and incubated for 5 minutes with $5 \mu \mathrm{g} / \mathrm{ml}$ Hoechst 33342 (Molecular
Probes) and analyzed by confocal microscopy (Nikon A1). Only sections containing the optic nerve were included for analysis. The density of cone nuclei was quantified manually on each section. Five sections through the optic nerve were collected and averaged for each mouse.

$E R G$. After mice were dark adapted overnight, recordings were obtained under dim red light illumination. Mice were anesthetized with an anesthetic solution ( $1 \mathrm{ml}$ of $100 \mathrm{mg} / \mathrm{ml} \mathrm{ketamine} \mathrm{and} 0.1 \mathrm{ml}$ of $20 \mathrm{mg}$ / $\mathrm{ml}$ xylazine in $8.9 \mathrm{ml} \mathrm{PBS}$ ) at a concentration of $0.1 \mathrm{ml} / 10 \mathrm{~g}$ BW injected in the intraperitoneal region. Heating pads were used to maintain body temperature at $37^{\circ} \mathrm{C}$. One drop of Tropicamide Ophthalmic Solution (1\%, Akorn) was administered in each eye for dilation. Ten minutes later, electrodes were placed on the corneas, and Goniosol hypromellose ophthalmic demulcent solution (2.5\%, Akron) was applied.

Both eyes were recorded simultaneously. Electrophysiological system (Diagnosys) was used to record ERG responses concurrently from both eyes. For rod and maximal rod and cone ERG responses, pulses of $0.00130 \mathrm{~cd} / \mathrm{m}^{2}$ and $3 \mathrm{~cd} / \mathrm{m}^{2}$ (White-6500K) were used. Each result represents the average of 40-60 trials. For cone responses, mice were light adapted in the Ganzfeld dome for 10 minutes. A background of $30 \mathrm{~cd} / \mathrm{m}^{2}$ (White-6500K) was present throughout the trials to suppress rod function. ERGs were recorded using white flashes. ERGs were recorded at 4-8 and 10 weeks.

Subretinal AAV injections. The AAV2/8(Y733F)-Sirt6_shRNA virus was injected subretinally at $\mathrm{P} 4$ in $P d e 6 b^{\mathrm{H} 620 \mathrm{Q} / \mathrm{H} 620 \mathrm{O}}$ mice. The eyelids of one eye of the newborn mouse were opened artificially using microsurgery scissors. AAV was transduced into the dorsal retinae by subretinal injection. Dorsal retinae of the right eyes were injected with AAV2/8(Y733F)-Sirt6_shRNA vector, whereas the left eyes were injected with same dose of PBS. The ventral side of all eyes was left untreated. Mice were examined by direct ophthalmoscopy to rule out retinal detachment at P21. ERG and histology data were collected at 4 weeks of age.

Three isoforms of PFK shRNA lentiviral particles were purchased from Sigma-Aldrich (ID number: GFP: MFCD07785395, PFK-M:TRCN0000319795, PFK-L:TRCN0000360434, PFKP:TRCN0000274701). Lentivirus was injected subretinally at P4-P6 in Pde6b ${ }^{H 620 Q / H 620 Q}$ mice. The same injection method was used as in the Sirt6 AAV injection described above. ERG data were collected at 3 weeks of age, while histology samples were collected at 4 weeks of age at $0.3 \mathrm{~mm}$ distal from the optic nerve head.

${ }^{13} \mathrm{C}$-labeled isotopetracingand mass spectrometry. ${ }^{13} \mathrm{C}$-labeled D-glucose $\left(\mathrm{U}-{ }^{13} \mathrm{C}_{6}, 99 \%\right)$ was purchased from Cambridge Isotope Laboratories Inc.

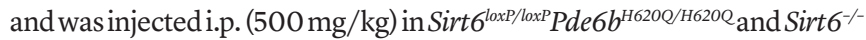
$P d e 6 b^{\mathrm{H} 620 \mathrm{O} / \mathrm{H} 620 \mathrm{O}}$ mice. After 45 minutes, retinae were promptly isolated from the mice, rinsed in PBS, and flash-frozen in liquid nitrogen. The retinae were subsequently homogenized in a mixture of methanol, chloroform, and water (700:200:50). The metabolites were dried, derivatized, and analyzed by GC-MS (Agilent 7890/5975C) as previously reported $(95,96)$. The chromatograms were analyzed using Agilent ChemStation software. The measured distribution of mass isotopologues was corrected for based on the natural abundance of isotopes using IsoCor software. The fractional abundance of labeled ions to total ion intensity was determined. Data were collected for Sirt $6^{\text {loxP } / 0 \times P} P d e 6 b^{H 620 Q / H 620 Q}$ and Sirt6 ${ }^{-/}$Pde $6 b^{\mathrm{H} 620 \mathrm{Q} / \mathrm{H} 620 \mathrm{Q}}$ mice at P21 and P28.

Steady-state metabolites were measured by LC-MS as previously reported by Du et al. (95). Three-week-old control and experimental mice were sacrificed, and retinae were collected, rinsed in PBS, and 
flash-frozen in liquid nitrogen. Retinae were harvested at each time point, and metabolites extracted in cold $80 \%$ methanol and quantified with an Agilent 1260 LC (Agilent Technologies)-AB Sciex QTrap 5500 mass spectrometer (AB Sciex) system.

Statistics. All data were analyzed using Excel (Microsoft), Stata 12.1 (StatCorp), and R 3.1.1 (https://www.r-project.org/). Mice were divided into 2 groups: control (Sirt6 ${ }^{\left.\text {loxP/loxP } P d e 6 b^{H 620 Q / H 620 Q}\right)}$ and Sirt6deficient (Sirt6 $/$ Pde6b $\left.b^{\mathrm{H} 620 \mathrm{Q} / \mathrm{H} 620 \mathrm{Q}}\right)$. ERG outcomes were measured over time for between-group comparison at specific time points. For analyses comparing groups at a fixed time point, linear mixed models with random intercepts were fit to the data because each mouse contributes 2 data points (one per eye). ERG measurements were used as outcome measurements, and the assigned group was the predictor.

The trajectory of ERG outcomes was also compared between groups using data from all time points. Linear mixed models were fit as before, but the predictors in this model were group, time, time squared, and interaction terms for group, time and group, and time squared. The quadratic component was included after graphically examining the individual mouse trajectories. This model was compared to a model without interaction terms using a likelihood ratio test to determine whether the groups had different trajectories.

For analyses of IS/OS thickness and ONL nuclei density in $\mathrm{H} \& \mathrm{E}$ sections, and for rhodopsin OS width and S-cone opsin OS width, sections from mouse retinae were taken at each time point, and multiple images of each section were collected and averaged. Each mouse only contributed one observation, so observations were considered independent. Two-tailed $t$ tests were used to compare controls with Sirt6-deficient mice, and $P$ values less than 0.05 were considered significant.

Image J analysis software was used to analyze bands in Western blots. Protein levels were normalized with respect to actin levels.

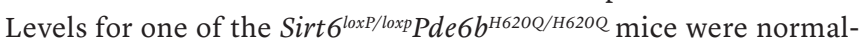
ized to 1 , and all other experimental conditions were compared with this. A $t$ test was used to compare the statistical difference between the groups.

Ten $\mathrm{C}^{13}$-labeled metabolites were measured to ascertain the ratio of labeled metabolite abundance to innate abundance, and these values were plotted. The abundance of glucose metabolites was also assessed by LC-MS. Data were $\log _{10}$ transformed and normalized to actin levels. Levels of the most statistically significantly changed metabolites involved in the glycolysis pathway were measured and plotted based on abundance. To compare groups for the previously mentioned outcomes, a two-sample $t$ test was used. To compare injection of AAV2/8(Y733F)-Sirt6_shRNA vector versus PBS, and the PFK virus injection versus GFP injection, a paired design was used, where the right eye of a mouse was injected with vector and the left with PBS. Then mixed ERG b-wave values were recorded and compared using a paired $t$ test.

Throughout all figures, error bars represent SD.

Study approval. The IACUC of Columbia University approved all experiments prior to study start. Use of mice was in accordance with the Statement for the Use of Animals in Ophthalmic and Vision Research of the Association for Research in Vision and Ophthalmology and the Policy for the Use of Animals in Neuroscience Research of the Society for Neuroscience.

\section{Author contributions}

All authors contributed equally to the data acquisition, analysis, and preparation of the manuscript.

\section{Acknowledgments}

The Jonas Children's Vision Care and Bernard \& Shirlee Brown Glaucoma Laboratory are supported by the NIH (5P30EY019007, R01EY018213, R01EY024698, 1R01EY026682, R21AG050437), National Cancer Institute Core (5P30CA013696), the Research to Prevent Blindness (RPB) Physician-Scientist Award, and unrestricted funds from RPB. SHT is a member of the RD-CURE Consortium and is supported by the Tistou and Charlotte Kerstan Foundation, the Schneeweiss Stem Cell Fund, New York State (C029572), the Foundation Fighting Blindness New York Regional Research Center Grant (C-NY05-0705-0312), and the Gebroe Family Foundation. JBH is supported by NIH (1R01EY02602001). VBM is supported by NIH (1R01EY026682, 5R21AG050437). Special thanks go to Amy Caudy, Eyal Gottlieb, and Adam Rosebrock for their consultations during the metabolomics course at Cold Spring Harbor Laboratory.

Address correspondence to: Stephen H. Tsang, 635 West 165th Street, Box 112, Columbia University Medical Center, New York, New York 10032, USA. Phone: 212.342.1186; E-mail: sht2@ columbia.edu.
1. Hamel C. Retinitis pigmentosa. Orphanet J Rare Dis. 2006;1:40.

2. Davis RJ, et al. Therapeutic margins in a novel preclinical model of retinitis pigmentosa. $\mathrm{J} \mathrm{Neu}$ rosci. 2013;33(33):13475-13483.

3. Shen S, Sujirakul T, Tsang SH. Next-generation sequencing revealed a novel mutation in the gene encoding the beta subunit of rod phosphodiesterase. Ophthalmic Genet. 2014;35(3):142-150.

4. Wu WH, et al. CRISPR repair reveals causative mutation in a preclinical model of retinitis pigmentosa. Mol Ther. 2016;24(8):1388-1394.

5. Tsang SH, et al. Role for the target enzyme in deactivation of photoreceptor $G$ protein in vivo. Science. 1998;282(5386):117-121.

6. Tsang SH, et al. Retinal degeneration in mice lacking the gamma subunit of the rod cGMP phosphodiesterase. Science. 1996;272(5264):1026-1029.

7. Wert KJ, Davis RJ, Sancho-Pelluz J, Nishina PM, Tsang SH. Gene therapy provides long-term visual function in a pre-clinical model of retinitis pigmentosa. Hum Mol Genet. 2013;22(3):558-567.

8. Wert KJ, Lin JH, Tsang SH. General pathophysiology in retinal degeneration. Dev Ophthalmol. 2014;53:33-43.

9. Dryja TP, Rucinski DE, Chen SH, Berson EL. Frequency of mutations in the gene encoding the alpha subunit of rod cGMP-phosphodiesterase in autosomal recessive retinitis pigmentosa. Invest Ophthalmol Vis Sci. 1999;40(8):1859-1865.

10. Davis RJ, et al. Functional rescue of degenerating photoreceptors in mice homozygous for a hypomorphic cGMP phosphodiesterase $6 \mathrm{~b}$ allele (Pde6bH620Q). Invest Ophthalmol Vis Sci. 2008;49(11):5067-5076.

11. Punzo C, Kornacker K, Cepko CL. Stimulation of the insulin/mTOR pathway delays cone death in a mouse model of retinitis pigmentosa. $\mathrm{Nat} \mathrm{Neu}$ rosci. 2009;12(1):44-52.

12. Venkatesh A, Ma S, Le YZ, Hall MN, Rüegg MA, Punzo C. Activated mTORC1 promotes longterm cone survival in retinitis pigmentosa mice. JClin Invest. 2015;125(4):1446-1458.

13. Nelson DL, Cox MM. Lehninger Principles of Biochemistry. New York, New York:W.H. Freeman; 2013.

14. Du J, et al. Phototransduction influences metabolic flux and nucleotide metabolism in mouse retina. J Biol Chem. 2016;291(9):4698-4710.

15. Okawa H, Sampath AP, Laughlin SB, Fain GL. ATP consumption by mammalian rod photo- 
receptors in darkness and in light. Curr Biol. 2008;18(24):1917-1921.

16. Nakanishi S. Second-order neurones and receptor mechanisms in visual- and olfactory-information processing. Trends Neurosci. 1995;18(8):359-364.

17. Linsenmeier RA. Effects of light and darkness on oxygen distribution and consumption in the cat retina. J Gen Physiol. 1986;88(4):521-542.

18. Ahmed J, Braun RD, Dunn R, Linsenmeier RA. Oxygen distribution in the macaque retina. Invest Ophthalmol Vis Sci. 1993;34(3):516-521.

19. Birol G, Wang S, Budzynski E, WangsaWirawan ND, Linsenmeier RA. Oxygen distribution and consumption in the macaque retina. Am J Physiol Heart Circ Physiol. 2007;293(3):H1696-H1704.

20. Du J, et al. Phototransduction influences metabolic flux and nucleotide metabolism in mouse retina. J Biol Chem. 2016;291(9):4698-4710.

21. Fariss RN, Li ZY, Milam AH. Abnormalities in rod photoreceptors, amacrine cells, and horizontal cells in human retinas with retinitis pigmentosa. Am JOphthalmol. 2000;129(2):215-223.

22. Curcio CA, Sloan KR, Kalina RE, Hendrickson AE. Human photoreceptor topography. JComp Neurol. 1990;292(4):497-523.

23. Maguire AM, et al. Safety and efficacy of gene transfer for Leber's congenital amaurosis. N Engl JMed. 2008;358(21):2240-2248.

24. Ashtari M, et al. Plasticity of the human visual system after retinal gene therapy in patients with Leber's congenital amaurosis. Sci Transl Med. 2015;7(296):296ra110.

25. Bainbridge JW, et al. Effect of gene therapy on visual function in Leber's congenital amaurosis. N Engl JMed. 2008;358(21):2231-2239.

26. Cideciyan AV, et al. Human RPE65 gene therapy for Leber congenital amaurosis: persistence of early visual improvements and safety at 1 year. Hum Gene Ther. 2009;20(9):999-1004.

27. Maguire AM, et al. Age-dependent effects of RPE65 gene therapy for Leber's congenital amaurosis: a phase 1 dose-escalation trial. Lancet. 2009;374(9701):1597-1605.

28. Simonelli F, et al. Gene therapy for Leber's congenital amaurosis is safe and effective through 1.5 years after vector administration. Mol Ther. 2010;18(3):643-650.

29. Jacobson SG, et al. Gene therapy for Leber congenital amaurosis caused by RPE 65 mutations: safety and efficacy in 15 children and adults followed up to 3 years. Arch Ophthalmol. 2012;130(1):9-24.

30. Bennett J, et al. AAV2 gene therapy readministration in three adults with congenital blindness. Sci Transl Med. 2012;4(120):120ra15.

31. Ashtari M, et al. The human visual cortex responds to gene therapy-mediated recovery of retinal function. J Clin Invest. 2011;121(6):2160-2168.

32. Testa F, et al. Three-year follow-up after unilateral subretinal delivery of adeno-associated virus in patients with Leber congenital amaurosis type 2. Ophthalmology. 2013;120(6):1283-1291.

33. Amado D, et al. Safety and efficacy of subretinal readministration of a viral vector in large animals to treat congenital blindness. Sci Transl Med. 2010;2(21):21ra16.

34. Vandenberghe LH, et al. Dosage thresholds for
AAV2 and AAV8 photoreceptor gene therapy in monkey. Sci Transl Med. 2011;3(88):88ra54.

35. Daiger SP, Bowne SJ, Sullivan LS. Perspective on genes and mutations causing retinitis pigmentosa. Arch Ophthalmol. 2007;125(2):151-158.

36. Lin M, Kim SH, Zhang L, Tsai Y, Tsang SH. Rod metabolic demand drives progression in retinopathies. Taiwan J Ophthalmol. 2015;5(3):105-108.

37. Yau KW. Phototransduction mechanism in retinal rods and cones. The Friedenwald Lecture. Invest Ophthalmol Vis Sci. 1994;35(1):9-32.

38. Zhang L, et al. Reprogramming towards anabolism impedes degeneration in a preclinical model of retinitis pigmentosa [published online ahead of print August 11, 2016]. Hum Mol Genet. doi:10.1093/hmg/ddw256.

39. Tsang SH, et al. Silencing of tuberin enhances photoreceptor survival and function in a preclinical model of retinitis pigmentosa (an american ophthalmological society thesis). Trans Am Ophthalmol Soc. 2014;112:103-115.

40. Martinez-Pastor B, Mostoslavsky R. Sirtuins, metabolism, and cancer. Front Pharmacol. 2012;3:22.

41. Zhong L, et al. The histone deacetylase Sirt6 regulates glucose homeostasis via Hiflalpha. Cell. 2010;140(2):280-293.

42. Zwaans BM, Lombard DB. Interplay between sirtuins, MYC and hypoxia-inducible factor in cancer-associated metabolic reprogramming. Di Model Mech. 2014;7(9):1023-1032.

43. Kleszcz R, Paluszczak J, Baer-Dubowska W. Targeting aberrant cancer metabolism - the role of sirtuins. Pharmacol Rep. 2015;67(6):1068-1080.

44. Etchegaray JP, Zhong L, Mostoslavsky R. The histone deacetylase SIRT6: at the crossroads between epigenetics, metabolism and disease. Curr Top Med Chem. 2013;13(23):2991-3000.

45. Silberman DM, et al. SIRT6 is required for normal retinal function. PLoS One. 2014;9(6):e98831.

46. Hahn LB, Berson EL, Dryja TP. Evaluation of the gene encoding the gamma subunit of rod phosphodiesterase in retinitis pigmentosa. Invest Ophthalmol Vis Sci. 1994;35(3):1077-1082.

47. Huang SH, Pittler SJ, Huang X, Oliveira L, Berson EL, Dryja TP. Autosomal recessive retinitis pigmentosa caused by mutations in the alpha subunit of rod cGMP phosphodiesterase. Nat Genet. 1995;11(4):468-471.

48. Danciger M, et al. Mutations in the PDE6B gene in autosomal recessive retinitis pigmentosa. Genomics. 1995;30(1):1-7.

49. Valvona CJ, Fillmore HL, Nunn PB, Pilkington GJ The regulation and function of lactate dehydrogenase a: therapeutic potential in brain tumor. Brain Pathol. 2016;26(1):3-17.

50. Fan J, et al. Tyrosine phosphorylation of lactate dehydrogenase A is important for $\mathrm{NADH} /$ $\mathrm{NAD}(+)$ redox homeostasis in cancer cells. $\mathrm{Mol}$ Cell Biol. 2011;31(24):4938-4950.

51. Uyeda K. Phosphofructokinase. Adv Enzymol Relat Areas Mol Biol. 1979;48:193-244.

52. Dimauro S, Akman O, Hays AP. Disorders of carbohydrate metabolism. Handb Clin Neurol. 2007;86:167-182.

53. Bramall AN, Wright AF, Jacobson SG, McInnes RR. The genomic, biochemical, and cellular responses of the retina in inherited photore- ceptor degenerations and prospects for the treatment of these disorders. Annu Rev Neurosci. 2010;33:441-472.

54. Veleri S, Lazar CH, Chang B, Sieving PA, Banin E, Swaroop A. Biology and therapy of inherited retinal degenerative disease: insights from mouse models. Dis Model Mech. 2015;8(2):109-129.

55. Yuzawa M, Fujita K, Tanaka E, Wang EC. Assessing quality of life in the treatment of patients with age-related macular degeneration: clinical research findings and recommendations for clinical practice. Clin Ophthalmol. 2013;7:1325-1332.

56. Daiger SP, Sullivan LS, Gire AI, Birch DG, Heckenlively JR, Bowne SJ. Mutations in known genes account for $58 \%$ of autosomal dominant retinitis pigmentosa (adRP). Adv Exp Med Biol. 2008;613:203-209.

57. Zhang Q. Retinitis pigmentosa: progress and perspective. Asia Pac JOphthalmol (Phila). 2016;5(4):265-271.

58. Sorrentino FS, Gallenga CE, Bonifazzi C, Perri P. A challenge to the striking genotypic heterogeneity of retinitis pigmentosa: a better understanding of the pathophysiology using the newest genetic strategies [published online ahead of print August, 26, 2016]. Eye (Lond). doi: 10.1038/eye.2016.197.

59. Bainbridge JW, et al. Long-term effect of gene therapy on Leber's congenital amaurosis. $N$ Engl J Med. 2015;372(20):1887-1897.

60. Solinís MÁ, del Pozo-Rodríguez A, Apaolaza PS, Rodríguez-Gascón A. Treatment of ocular disorders by gene therapy. Eur J Pharm Biopharm. 2015;95(Pt B):331-342.

61. Constable IJ, Blumenkranz MS, Schwartz SD, Barone S, Lai CM, Rakoczy EP. Gene therapy for age-related macular degeneration. Asia Pac J Ophthalmol (Phila). 2016;5(4):300-303.

62. Ginn SL, Alexander IE, Edelstein ML, Abedi MR, Wixon J. Gene therapy clinical trials worldwide to 2012 - an update. J Gene Med. 2013;15(2):65-77.

63. MacLaren RE, et al. Retinal gene therapy in patients with choroideremia: initial findings from a phase $1 / 2$ clinical trial. Lancet. 2014;383(9923):1129-1137.

64. Trapani I, Banfi S, Simonelli F, Surace EM, Auricchio A. Gene therapy of inherited retinal degenerations: prospects and challenges. Hum Gene Ther. 2015;26(4):193-200.

65. Hurley JB, Lindsay KJ, Du J. Glucose, lactate, and shuttling of metabolites in vertebrate retinas. J Neurosci Res. 2015;93(7):1079-1092.

66. Vander Heiden MG, Cantley LC, Thompson CB. Understanding the Warburg effect: the metabolic requirements of cell proliferation. Science. 2009;324(5930):1029-1033.

67. Vazquez A, Liu J, Zhou Y, Oltvai ZN. Catabolic efficiency of aerobic glycolysis: the Warburg effect revisited. BMC Syst Biol. 2010;4:58.

68. Chen B, Cepko CL. HDAC4 regulates neuronal survival in normal and diseased retinas. Science. 2009;323(5911):256-259.

69. Aït-Ali N, et al. Rod-derived cone viability factor promotes cone survival by stimulating aerobic glycolysis. Cell. 2015;161(4):817-832.

70. Wise DR, et al. Myc regulates a transcriptional program that stimulates mitochondrial glutaminolysis and leads to glutamine addiction. Proc 
Natl Acad Sci U S A. 2008;105(48):18782-18787.

71. Maher EA, et al. Metabolism of [U-13 C]glucose in human brain tumors in vivo. NMR Biomed. 2012;25(11):1234-1244.

72. Buescher JM, et al. A roadmap for interpreting (13)C metabolite labeling patterns from cells. Curr Opin Biotechnol. 2015;34:189-201.

73. Yang Y, et al. Functional cone rescue by RdCVF protein in a dominant model of retinitis pigmentosa. Mol Ther. 2009;17(5):787-795.

74. Léveillard T, Sahel JA. Rod-derived cone viability factor for treating blinding diseases: from clinic to redox signaling. Sci Transl Med. 2010;2(26):26ps16.

75. Léveillard T, et al. Identification and characterization of rod-derived cone viability factor. Nat Genet. 2004;36(7):755-759.

76. Mohand-Said S, et al. Normal retina releases a diffusible factor stimulating cone survival in the retinal degeneration mouse. Proc Natl Acad Sci USA. 1998;95(14):8357-8362.

77. Mohand-Said S, Hicks D, Dreyfus H, Sahel JA. Selective transplantation of rods delays cone loss in a retinitis pigmentosa model. Arch Ophthalmol. 2000;118(6):807-811.

78. Xiong W, MacColl Garfinkel AE, Li Y, Benowitz LI, Cepko CL. NRF2 promotes neuronal survival in neurodegeneration and acute nerve damage. J Clin Invest. 2015;125(4):1433-1445.

79. Cepko C, Punzo C. Cell metabolism: sugar for sight. Nature. 2015;522(7557):428-429.

80. Cideciyan AV, et al. Human retinal gene therapy for Leber congenital amaurosis shows advancing retinal degeneration despite enduring visual improvement. Proc Natl Acad Sci U S A. 2013;110(6):E517-E525.

81. Jacobson SG, et al. Improvement and decline in vision with gene therapy in childhood blindness. N Engl J Med. 2015;372(20):1920-1926.

82. Wright AF. Long-term effects of retinal gene therapy in childhood blindness. N Engl JMed. 2015;372(20):1954-1955.

83. Demetrius LA, Magistretti PJ, Pellerin L. Alzheimer's disease: the amyloid hypothesis and the inverse Warburg effect. Front Physiol. 2014;5:522.

84. Demetrius LA, Simon DK. An inverse-Warburg effect and the origin of Alzheimer's disease. Biogerontology. 2012;13(6):583-594.

85. Mariani AP. Neuronal and synaptic organization of the outer plexiform layer of the pigeon retina. Am J Anat. 1987;179(1):25-39.

86. Masland RH. The neuronal organization of the retina. Neuron. 2012;76(2):266-280.

87. Kim HS, et al. Hepatic-specific disruption of SIRT6 in mice results in fatty liver formation due to enhanced glycolysis and triglyceride synthesis. Cell Metab. 2010;12(3):224-236.

88. Hart AW, et al. Genotype-phenotype correlation of mouse pde6b mutations. Invest Ophthalmol Vis Sci. 2005;46(9):3443-3450.
89. Koch SF, et al. Halting progressive neurodegeneration in advanced retinitis pigmentosa. JClin Invest. 2015;125(9):3704-3713.

90. Tsang SH, et al. Retarding photoreceptor degeneration in Pdegtm1/Pdegtml mice by an apoptosis suppressor gene. Invest Ophthalmol Vis Sci. 1997;38(5):943-950.

91. Tsang SH, et al. GAP-independent termination of photoreceptor light response by excess gamma subunit of the cGMP-phosphodiesterase. J Neurosci. 2006;26(17):4472-4480.

92. Tosi J, Davis RJ, Wang NK, Naumann M, Lin CS, Tsang SH. shRNA knockdown of guanylate cyclase 2e or cyclic nucleotide gated channel alpha 1 increases photoreceptor survival in a cGMP phosphodiesterase mouse model of retinitis pigmentosa. JCell Mol Med. 2011;15(8):1778-1787.

93. Tsang SH, et al. A novel mutation and phenotypes in phosphodiesterase 6 deficiency. Am JOphthalmol. 2008;146(5):780-788.

94. Tsang SH, et al. Effect of the ILE86TER mutation in the $\gamma$ subunit of cGMP phosphodiesterase (PDE6) on rod photoreceptor signaling. Cell Signal. 2012;24(1):181-188.

95. Du J, Linton JD, Hurley JB. Probing metabolism in the intact retina using stable isotope tracers. Meth Enzymol. 2015;561:149-170.

96. Du J, et al. Cytosolic reducing power preserves glutamate in retina. Proc Natl Acad Sci US A. 2013;110(46):18501-18506. 